\title{
Reliability-based design optimization of composite stiffened panels in post-buckling regime
}

\author{
Carlos López · Omar Bacarreza • Aitor \\ Baldomir • Santiago Hernández • M.H. \\ Ferri Aliabadi
}

Received: / Accepted:

\begin{abstract}
This paper focuses on Deterministic and Reliability Based Design Optimization (DO and RBDO) of composite stiffened panels considering post-buckling regime and progressive failure analysis. The ultimate load that a post-buckled panel can hold is to be maximised by changing the stacking sequence of both skin and stringers composite layups. The RBDO problem looks for a design that collapses beyond the shortening of failure obtained in the DO phase with a target reliability while considering uncertainty in the elastic properties of the composite material. The RBDO algorithm proposed is decoupled and hence separates the Reliability Analysis (RA) from the deterministic optimization. The main code to drive both the DO and RBDO approaches is written in MATLAB and employs Genetic Algorithms (GA) to solve the DO loops because discrete design variables and highly nonlinear response functions are expected. The code is linked with Abaqus to perform parallel explicit nonlinear finite element analyses in order to obtain the structural responses at each generation. The RA is solved through an inverse Most Probable failure Point (MPP) search algorithm that benefits from a Poly-
\end{abstract}

Carlos López · Aitor Baldomir · Santiago Hernández

Structural Mechanics Group. School of Civil Engineering, Universidade da Coruña,

Campus de Elviña s/n, 15071 A Coruña, Spain

E-mail: carlos.lopez.rodriguez@udc.es

Aitor Baldomir

E-mail: abaldomir@udc.es

Santiago Hernández

E-mail: hernandez@udc.es

Omar Bacarreza - M.H. Ferri Aliabadi

Department of Aeronautics, Imperial College London,

London, SW7 2AZ, UK

E-mail: o.bacarreza-nogales@imperial.ac.uk

M.H. Ferri Aliabadi

E-mail: m.h.aliabadi@imperial.ac.uk 
nomial Chaos Expansion with Latin Hypercube Sampling (PCE-LHS) metamodel when the structural responses are required. The results led to small reductions in the maximum load that the panels can bear but otherwise assure that they will collapse beyond the shortening of failure imposed with a high reliability.

Keywords Reliability · optimization · composites · stiffened panel · progressive failure

\section{Introduction}

Aerospace companies are increasingly demanding lighter, safer and cleaner aircrafts, being at the spearhead of innovation in the use of composite materials. They are already playing a major role in the construction of aeronautical components that takes part of larger structures like the fuselage, the wings or both the vertical and horizontal tail plane (VTP-HTP). For example, the Boeing 787 Dreamliner has a $50 \%$ of its structure built of composite materials (Hawk (2005)), whereas the new Airbus A350 XWB reaches the 52\% (Marsh (2007)). These percentages do nothing but grow as they maximise weight reduction (typically $20 \%$ lighter than aluminum), add strength, increase the lifespan of the aircraft thanks to a superior durability and reduce maintenance costs as they require a lower number of inspections during service. By constrast, composite materials lack the well-established industrial manufacture processes of the traditional metals and alloys, which involve a higher uncertainty when dealing with their mechanical properties in structural tests or analyses.

Moreover, besides the uncertainty associated to the composite mechanical properties there may be other sources of error in load values, structural sizes or manufacturing processes. In the design of an aircraft engineers need to take into account this uncertainty in order to meet the severe design conditions imposed by international regulations. Traditionally this was accomplished by imposing safety factors during the design process that are usually based on experience. The novel approach to consider uncertainty in aeronautical structures is to perform Reliability Analysis (RA) and Reliability-Based Design Optimization (RBDO) during the design process.

It is usual that aerospace companies try to produce derivatives from previous products, namely aircrafts, trying to increase their efficiency by evaluating how they can endure higher values of loads. The main structures of an aircraft are usually made of stiffened panels, which are designed for maximum strength, stiffness and buckling load. However, it is well-known that the buckling load does not represent the maximum load that the structure can bear, indeed it may hold several times the first buckling load withouth any damage (Stevens et al (1995), Zimmermann and Rolfes (2006), Degenhardt et al (2006)). Consequently some panels are designed to work in the post-buckling regime due to their high slenderness and that the post-buckling extra strength has potential to achieve lighter and more economic designs.

The widespread use of stiffened panels as structural components leads to use Deterministic Optimization (DO) processes to achieve the best possible design. Performing an optimization problem for the design of stiffened composite panels may be troublesome given that several variables of different natures may be 
involved, as geometric dimensions or stacking sequences of the composite layup. In addition, the behavior of the structure is highly nonlinear being largely conditioned by the buckling phenomenon and the demanding aeronautical industry advocates the study of post-buckling regime, where the nonlinearities are even of higher order. For those reasons Genetic Algorithms (GA) are commonly used to deal with these problems since they can handle discrete, continuous and mixed design variables and are effective with nonlinear and non-convex functions. GAs are based on the Darwin's "natural selection" principle (Goldberg (1989), Holland (1992)) and are translated to mathematical terminology through the creation of a population of individuals that evolve and improve thanks to the operators of crossover, mutation and selection. Some other population-based algorithms such as the particle swarm algorithm have shown effectiveness in the optimization of composite materials with high nonlinearities (Xu et al (2012), Xu and You (2013), Shabana and Elsawaf (2015)).

In engineering, the evaluation of the objective function and constraints is usually performed through a nonlinear Finite Element (FE) analysis. Since the GA are population-based methods that require a large number of evaluations of the objective function and constraints before reaching the optimum, the solution of the optimization problem involves a high computational effort that may become prohibitive for industrial applications. Otherwise dealing with several discrete design variables increases the number of possible configurations, making the whole design space almost boundless. For example, Faggiani and Falzon (2007) minimized the damage between the skin and stiffeners of a composite panel in post-buckling, which required 18 generations of 40 individuals (720 FE analyses). Badalló et al (2013) made a comparative study of three different GAs in a multiobjective optimization problem that required 25 generations of 16 individuals (400 FE analyses) for each case.

To overcome the computational difficulties associated with this kind of optimization problems, another strategy increasingly used is based on global approximation techniques or metamodel regressions (Kriging models, Artificial Neural Networks, Radial Basis Functions, Polynomial Chaos Expansion or Multivariate Adaptative Regression Splines are some examples). There are several studies where different metamodel regression methods are compared for a wide range of applications (Jin et al (2001), Acar and Rais-Rohani (2009), Simpson et al (2014)). From a limited number of FE simulation samples they build a metamodel that captures the behavior of the overall design region. Then the GA is performed over the metamodel instead of running the FE analyses required for each generation. Some examples of these strategies to optimize stiffened panels in both buckling and post-buckling regime are presented in Todoroki and Sekishiro (2008), Bisagni and Lanzi (2002), Lanzi and Giavotto (2006), Irisarri et al (2011) or Marín et al (2012). One of the main limitations of global approximation methods is that it is impossible to identify a priori which approximation technique is the best (Jin et al (2001)). Moreover, in some cases the number of FE simulations required to obtain a good approximation may be elevated and therefore the computational effort saving with respect to GA with FE analyses is reduced.

Otherwise, RA predicts the probability of failure of a determined limit-state of a structure by considering the real distribution functions of the uncertain data assuming that they are random variables. Several techniques for performing RA have been developed including moment-based methods, sampling methods or approxi- 
mation techniques (Choi et al (2007), Zhao and Ono (2001), Eldred et al (2007) or Choi et al (2004)). There is some research on RA applied to stiffened panels. Akula (2014) performs a multiscale reliability analysis of a composite stiffened panels considering microscale and macroscale design parameters. The probability of failure is predicted using the Monte Carlo method in combination with approximation models. Rognin et al (2010) develops a simulation approach to enhance the reliability of complex damaged composite structures. Chen and Soares (2007) presents a method that couples FE analyses with an enhanced first-order reliability algorithm for reliability estimation of the post-buckling compressive strength of panels under compression loads.

RBDO aims to address the best possible design through optimization while taking into account the uncertain data in some parameters that affect the structural responses, seeking for the best compromise between cost and safety. This discipline requires high computational effort since it combines DO and RA and has been applied to several multidisciplinary areas as automotive (Sinha (2007), Youn et al (2004) or Cid Montoya et al (2015)), naval (Leheta and Mansour (1997)) or civil engineering (Karadeniz et al (2009) or Kusano et al (2015)). Talking about RBDO applied to aeronautical stiffened panels, Hernandez et al (2013) draws a comparison of three RBDO methods in a metallic panel under buckling constraints, Qu and Haftka (2003) performs RBDO using Monte Carlo Simulations (MCS) and a Design Response Surface (DRS) in order to compute the reliability constraints while Díaz et al (2016) compares stochastic expansions with moment-based methods in the RA and genetic with gradient-based algorithms in the DO phase.

The goal of this research is to perform the RBDO of a composite stiffened panel focusing on maximizing its bearing capacity for a prefixed geometry and setting the shortening of failure as the probabilistic constraint. For that, the design variables are the orientation of the plies while the random parameters are the elastic properties of the composite. In order to predict the collapse load properly, post-buckling and progressive damage analyses are considered as an intrinsic part of the problem. In this study the reference panel employed is obtained from the preliminary design optimization phase performed in Bacarreza et al (2015), where the number of plies, number of stiffeners and geometry dimensions were considered as design variables, being the targets to minimize the mass and the Tsai-Wu index and maximize the reaction force.

\section{Definition of the Reliability-Based Design Optimization problem}

2.1 Description of the stiffened panel and physics of the problem

This research employs the stiffened composite panel presented in Figure 1, which is defined by a flat skin with three stringers. These kind of panels are part of more complex aircraft structures like the wings or the fuselage. The panel is fixed at one of the transversal edges and loaded under compression in the opposite edge by a uniform increasing displacement that is applied in both the skin and stringers. The longitudinal edges are constrained in all degrees of freedom except in the x-direction (Figure 1). The overall dimensions of the panel are $L=1196.0 \mathrm{~mm}$ length and $W=618.4 \mathrm{~mm}$ width, while the geometric dimensions of the stringers are given in Figure 2 and Table 1. 


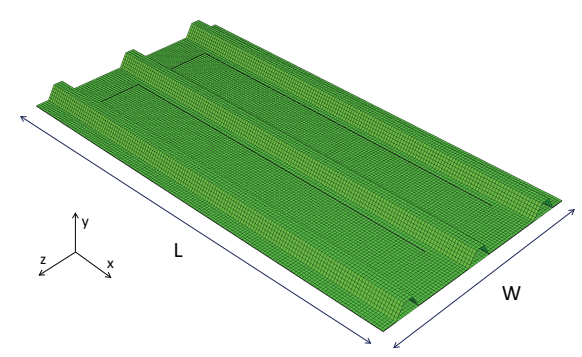

(a) Overall dimensions

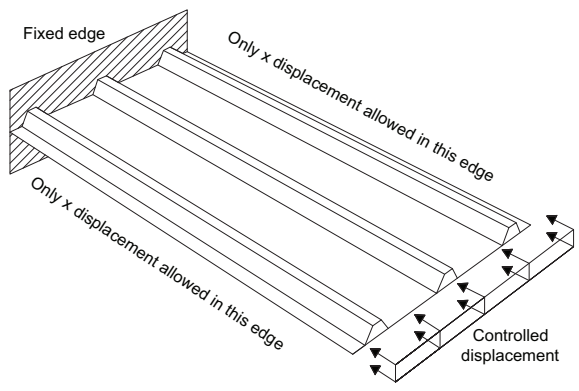

(b) Scheme of the numerical experiment

Fig. 1: Overall dimensions of the stiffened panel and scheme of the numerical experiment.

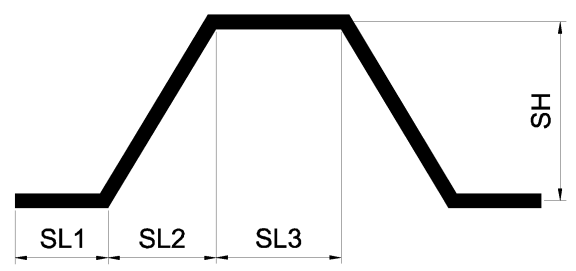

Fig. 2: Geometric dimensions of the stringers.

Table 1: Geometric dimensions of the stringers.

\begin{tabular}{lll}
\hline Dimension & Description & Value \\
\hline SH $[\mathrm{mm}]$ & Stringer Height & 37.46 \\
SL1 [mm] & Stringer Length 1 & 19.57 \\
SL2 [mm] & Stringer Length 2 & 22.77 \\
SL3 [mm] & Stringer Length 3 & 26.33 \\
\hline
\end{tabular}

The skin and stringers of the panel are built as separate composite pieces and a cohesive material is modelled as the interface between them. The densities of both materials are of $1550 \mathrm{~kg} / \mathrm{m}^{3}$ and $1600 \mathrm{~kg} / \mathrm{m}^{3}$ respectively, being their mechanical properties presented in Table 2 .

The skin of the panel is an 8-ply symmetric and balanced laminate with stacking sequence $[45,-45,0,90] \mathrm{s}$, whereas the stringers are constituted by a 10-ply symmetric and balanced laminate $[45,0,-45,0,90] \mathrm{s}$. The thickness of a single layer of the skin is $T_{s k}=0.276 \mathrm{~mm}$, while in the stringers it is $T_{s t}=0.186 \mathrm{~mm}$. This means that the total thicknesses of the skin and the stringers are $2.208 \mathrm{~mm}$ and $1.86 \mathrm{~mm}$, respectively. 
Table 2: Material properties.

\begin{tabular}{|c|c|c|}
\hline Property & Value & Description \\
\hline \multicolumn{3}{|c|}{ 1. Elastic properties of the composite material } \\
\hline$E_{11}[\mathrm{MPa}]$ & 159000.0 & Longitudinal modulus of elasticity \\
\hline$E_{22}=E_{33}[\mathrm{MPa}]$ & 10000.0 & Transversal modulus of elasticity \\
\hline$G_{23}[\mathrm{MPa}]$ & 5000.0 & Longitudinal shear modulus \\
\hline$G_{12}=G_{13}[\mathrm{MPa}]$ & 3000.0 & Transversal shear modulus \\
\hline$\nu_{12}=\nu_{13}$ & 0.3 & Longitudinal Poisson's ratio \\
\hline$\nu_{23}$ & 0.52 & Transversal Poisson's ratio \\
\hline \multicolumn{3}{|c|}{ 2. Damage initiation parameters of the composite material } \\
\hline$\sigma_{1}^{0 \mathrm{t}}[\mathrm{MPa}]$ & 2413.0 & Maximum longitudinal tensile stress \\
\hline$\sigma_{2}^{0 t}=\sigma_{3}^{0 t}[\mathrm{MPa}]$ & 59.0 & Maximum transversal tensile stress \\
\hline$\sigma_{1}^{0 c}[\mathrm{MPa}]$ & 1655.0 & Maximum longitudinal compressive stress \\
\hline$\sigma_{2}^{0 c}=\sigma_{3}^{0 c}[\mathrm{MPa}]$ & 186.0 & Maximum transversal compressive stress \\
\hline$\tau_{12}^{0}=\tau_{12}^{0}[\mathrm{MPa}]$ & 121.0 & Maximum longitudinal shear stress \\
\hline$\tau_{23}^{0}[\mathrm{MPa}]$ & 85.0 & Maximum transversal shear stress \\
\hline$G_{c 1}^{t}[\mathrm{~N} / \mathrm{mm}]$ & 110.0 & Longitudinal tensile fracture energy \\
\hline$G_{c 2}^{t}=G_{c 3}^{t}[\mathrm{~N} / \mathrm{mm}]$ & 0.25 & Transversal tensile fracture energy \\
\hline$G_{c 1}^{c}[\mathrm{~N} / \mathrm{mm}]$ & 90.0 & Longitudinal compressive fracture energy \\
\hline$G_{c 2}^{c}=G_{c 3}^{c}[\mathrm{~N} / \mathrm{mm}]$ & 0.80 & Transversal tensile fracture energy \\
\hline \multicolumn{3}{|c|}{ 3. Elastic properties of the cohesive material } \\
\hline$E_{m}[\mathrm{MPa}]$ & 4350.0 & Modulus of elasticity \\
\hline$\nu_{m}$ & 0.36 & Poisson's ration \\
\hline \multicolumn{3}{|c|}{ 4. Damage initiation parameters of the cohesive material } \\
\hline$\sigma_{m}^{0}[\mathrm{MPa}]$ & 69.0 & Maximum nominal normal stress \\
\hline$\tau_{m}^{0}[\mathrm{MPa}]$ & 80.0 & Maximum nominal shear stress \\
\hline$G_{m}^{t}[\mathrm{~N} / \mathrm{mm}]$ & 0.13 & Normal fracture energy \\
\hline$G_{m}^{s}[\mathrm{~N} / \mathrm{mm}]$ & 0.65 & Shear fracture energy \\
\hline
\end{tabular}

As exposed in Section 1, the post-buckling extra strength has potential in order to achieve safer and more economic designs. Nowadays the tendency in industry is to perform virtual testings (Ostergaard et al (2011)) of FE models and then calibrate them with a low number of experiments instead of carrying out a high number of experiments in order to save costs and resources. Virtual testing allows to predict the behaviour of the panel in service and extreme conditions with the idea of gradually starting to certify certain structural components based on the FE model behavior without the need of carrying out experimental tests. For this reason, detailed FE models need to be constructed and increasingly advanced FE analyses are required. In this research nonlinear explicit dynamic FE analyses are used for modelling the post-buckling behaviour and the progressive failure of the composite stiffened panel.

During post-buckling, the shape of the buckling waves does not remain constant when increasing the compressive load. Indeed abrupt changes may occur at certain load levels, which are known as mode-switchs. These phenomena are dynamic instabilities that cause numerical difficulties when using quasi-static analyses. For a better performance, explicit FE analyses can be used (Bisagni (2000)). Predicting 
the collapse load of the design is difficult because of the sensitivity of composites to the effect of through-thickness stresses and the variety of damage mechanisms that can arise in several locations of the panel and which could lead to collapse.

The intralaminar failure is modelled through cohesive elements and is analysed based on continuum damage mechanics, taking into account all the possible failure modes including tension or compression failure of the fibres, and cracking, crushing or shear failure of the matrix. The definition of the damage initiation criteria is based on the Hashin criteria (Hashin and Rotem (1973) and Hashin (1980)), while the damage propagation laws are driven by the amount of energy dissipated during the damage process (Lapczyk and Hurtado (2007)). The Hashin initiation criteria are expressed as:

$$
\begin{gathered}
\text { Fiber tension }\left(\hat{\sigma}_{11} \geq 0\right): F_{f}^{t}=\left(\frac{\hat{\sigma}_{11}}{X^{T}}\right)^{2}+\alpha\left(\frac{\hat{\tau}_{12}}{S^{L}}\right)^{2} . \\
\text { Fiber compression }\left(\hat{\sigma}_{11}<0\right): F_{f}^{c}=\left(\frac{\hat{\sigma}_{11}}{X^{C}}\right)^{2} . \\
\text { Matrix tension }\left(\hat{\sigma}_{22} \geq 0\right): F_{m}^{t}=\left(\frac{\hat{\sigma}_{22}}{Y^{T}}\right)^{2}+\left(\frac{\hat{\tau}_{12}}{S^{L}}\right)^{2} . \\
\text { Matrix comp }\left(\hat{\sigma}_{22}<0\right): F_{m}^{c}=\left(\frac{\hat{\sigma}_{22}}{2 S^{T}}\right)^{2}+\left[\left(\frac{Y^{C}}{2 S^{T}}\right)^{2}-1\right] \frac{\hat{\sigma}_{22}}{Y^{C}}+\left(\frac{\hat{\tau}_{12}}{S^{L}}\right)^{2} .
\end{gathered}
$$

where $X^{T}$ and $X^{C}$ are the longitudinal tensile and compressive strengths, $Y^{T}$ and $Y^{C}$ are the transverse tensile and compressive strengths, $S^{L}$ and $S^{T}$ the longitudinal and transverse shear strength, $\alpha$ is a coefficient that determines the contribution of the shear stress to the fiber tensile initiation criteria and $\hat{\sigma}_{11}, \hat{\sigma}_{22}$ and $\hat{\tau}_{12}$ are the components of the effective stress tensor $\hat{\sigma}$, which is employed to evaluate the initiation criteria and is expressed as:

$$
\hat{\sigma}=\mathbf{M} \sigma
$$

where $\sigma$ is the true stress and $\mathbf{M}$ is the damage operator:

$$
\mathbf{M}=\left[\begin{array}{ccc}
\frac{1}{1-d_{f}} & 0 & 0 \\
0 & \frac{1}{1-d_{m}} & 0 \\
0 & 0 & \frac{1}{1-d_{s}}
\end{array}\right]
$$

where $d_{f}, d_{m}$ and $d_{s}$ are the internal damage variables that characterize fiber, matrix and shear damage. When the damage has happened, the behaviour of the damaged material includes stiffness degradation and is computed as:

$$
\sigma=\mathbf{C}_{d} \varepsilon
$$

being $\varepsilon$ the strain and $\mathbf{C}_{d}$ the damaged elasticity matrix, which is expressed as follows:

$$
\mathbf{C}_{d}=\frac{1}{D}\left[\begin{array}{ccc}
\left(1-d_{f}\right) E_{1} & \left(1-d_{f}\right)\left(1-d_{m}\right) \nu_{12} E_{1} & 0 \\
\left(1-d_{f}\right)\left(1-d_{m}\right) \nu_{12} E_{1} & \left(1-d_{m}\right) E_{2} & 0 \\
0 & 0 & \left(1-d_{s}\right) G D
\end{array}\right]
$$


where $D=1-\left(1-d_{f}\right) \cdot\left(1-d_{m}\right) \cdot \nu_{12} \cdot \nu_{21}$. Prior to any damage initiation and evolution, the damage operator $\mathbf{M}$ is equal to the identity matrix, so $\hat{\sigma}=\sigma$.

In this study the FE model considering post-buckling analysis, progressive damage and failure is built and calculated using Abaqus Explicit 6.14.2 (Abaqus (2014)), where both the Hashin damage initiation criteria and damage evolution laws are already internally implemented. When any of the expressions of equation 1 is accomplished in a certain finite element, the internal damage variables $d_{f}, d_{m}$ and $d_{s}$ are activated leading to the damage initiation stage. These parameters change the damage operator $\mathbf{M}$ of equation 3, which becomes different than the identity matrix, and the damaged elasticity matrix $\mathbf{C}_{d}$ in order to simulate the response of the damaged material. The damage evolution process is determined by the energy dissipated in the failure event (fracture energy) since the initiation of damage has occurred.

In this software, if $\alpha=0$ and $S_{T}=Y_{C} / 2$ the initiation criteria is the one proposed in Hashin and Rotem (1973), while if $\alpha=1$ it is the one presented in Hashin (1980). As exposed in Abaqus (2014) once the damage initiation and evolution has occurred for at least one mode, the damage operator becomes significant in the criteria for damage initiation of other modes. Figure $3 \mathrm{a}$ and Figure $3 \mathrm{~b}$ show the deformed shape of the panel during the post-buckling regime and after failure.

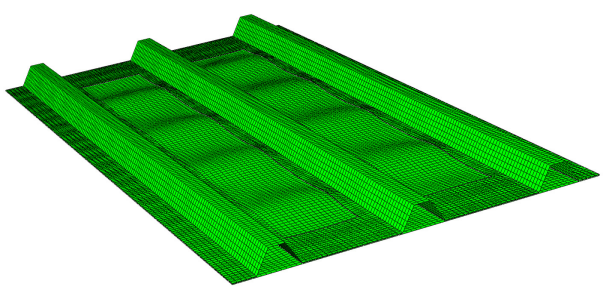

(a) Post-buckling

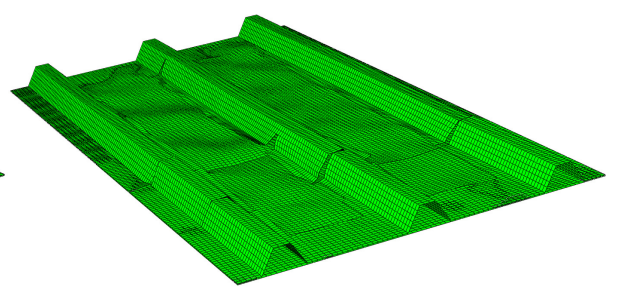

(b) After collapse

Fig. 3: Deformed shape of the panel in post-buckling regime (a) and after collapse (b).

Setting an optimization strategy for complex structures such as stiffened panels is a ticklish task, as there are several design variables of different nature involved (number of plies, number of stiffeners, thickness, dimensions of the panel and stiffeners, stacking orientations...) and several structural properties prone to be set as objective function and design constraints (mass, reaction force, buckling factor, shortening of collapse, Tsai-Wu index...). Some strategies tend to split the optimization process in several phases because including all that information in a single optimization problem may make it ungovernable. For instance, the optimization strategy proposed in Bacarreza et al (2015) divides the optimization process in two stages: a preliminary design optimization and a detailed design optimization.

The preliminary design optimization consists in performing an optimization where the mass and Tsai-Wu index try to be minimized and the reaction force maximized. In this phase the design variables considered are the number of plies, 
number of stiffeners and in general all the dimensions of the panel, being the material considered as linear elastic in order to shorten the FE simulation time.

Otherwise, the detailed design optimization takes the panel obtained in the previous phase and tries to maximize the bearing capacity considering as design variables the stacking orientations of the composite material, keeping the geometry and consequently the mass of the panel unaltered. In this stage, the post-buckling behaviour and progressive damage of the composite materials are considered in order to predict correctly the collapse load of the panel. These phenomena are influenced, among others, by the elastic properties of the materials. Since such properties are more prone to variations in composites than in other materiales, in this research they are considered as uncertain parameters.

It is important to highlight that the reference panel employed comes from the preliminary design optimization performed in Bacarreza et al (2015). Therefore the number of plies, thicknesses, number of stiffeners and geometry dimensions of the panel are optimum values already obtained from a previous optimization process aimed to minimize the mass of the structure. The motivation of this research is to carry out a RBDO of the detailed design optimization phase, assuming as design variables the stacking orientations of the plies, as random variables the elastic properties of the composites and considering the shortening of failure of the panel, which involves advanced FE simulations, as the probabilistic constraint. In other words, the target is to maximize the reaction force that the panel can support before collapse and at the same time assure with a target reliability that the collapse will happen beyond a certain value of shortening, which is obtained from a preliminary DO phase.

\subsection{Formulation of the deterministic optimization problem}

In the DO problem proposed, the geometry of the skin and stringers of the panel are fixed to the nominal configurations reported in Figure 1, Figure 2 and Table 1. The design variables of the problem are the stacking orientations of the layers that build the skin and stringers of the panel. Both layups are symmetric, which is usual in aerospace industry, so the number of design variables considered is 9 ( 4 in the skin and 5 in the stringers, which is half the number of layers affected). Each layer can take the orientation values of $-45^{\circ}, 0^{\circ}, 45^{\circ}$ or $90^{\circ}$ with respect to the longitunal axis of the panel, meaning that the design space is formed by $4^{9}=262144$ different designs. These orientation values are the guidelines employed in aerospace industry.

The fact that all the design variables are discrete and the FE model takes a long time to run a single FE analysis (parallelization is highly recommended) advocates the use of Genetic Algorithms (GA) to solve the optimization problem. GA excel in these two issues (dealing with integer or mixed design variables and parallelisation), although they are more design improvers rather than proper optimizers as they do not assure that the design obtained is an optimum. Besides, the convergence and performance of GA may depend on the parameters (number of individuals, number of generations) and operators of the algorithm.

In Bacarreza et al (2015) the problem was initially defined as a multi-objective optimization aimed to maximize both the reaction force $R f$ and the internal energy $E n$, subject to the fulfillment of a certain value of the reaction force $R f=655.0 \mathrm{kN}$. 
The optimization was carried out through the NGSA-II algorithm (Deb et al (2002)) and showed that the constraint was inactive and that the two objective functions followed a quasi linear relation. Afterwards in this research the DO problem was slightly modified turning it into unconstrained and mono-objective with the objective of just maximizing the reaction force $R f$ that the panel can hold before collapse, being the mathematical formulation of the problem presented below:

$$
\max R f
$$

As exposed in subsection 2.1, $R f$ is obtained through nonlinear explicit FE analyses performed in Abaqus 6.14.2 that involve using the damage initiation criteria and damage propagation laws of Eq. 1-5 within each simulation. Once the DO is completed the values of both the reaction force $R f$ and the internal energy $E n$ of the optimum panel are obtained. In this research three different configurations with a different number of generations (gen.) and individuals in the population (ind.) are tested for the same GA (10gen./30ind., 20gen./20ind. and 16gen./20ind.) and two different designs were found (Table 3 and Figure 4).

Table 3: Optimization results for different number of generations and individuals.

\begin{tabular}{lrrr}
\hline Description & Opt. des. 10gen./30ind. & Opt. des. 20gen./20ind. & Opt. des. 16gen./20ind. \\
\hline$R f[\mathrm{kN}]$ & 903.47 & 965.95 & 965.95 \\
$S h_{f}[\mathrm{~mm}]$ & 6.04 & 6.32 & 6.32 \\
$E n[\mathrm{~J}]$ & 3081.40 & 3402.21 & 3402.21 \\
Skin layup & {$[90,0,0,0] \mathrm{s}$} & {$[0,0,0,90] \mathrm{s}$} & {$[0,0,0,90] \mathrm{s}$} \\
Stringers layup & {$[90,-45,0,90,0] \mathrm{s}$} & {$[45,0,-45,0,0] \mathrm{s}$} & {$[45,0,-45,0,0] \mathrm{s}$} \\
\hline
\end{tabular}

In both 20gen./20ind. and 16gen./20ind. the convergence of the GA is clear since the value of the objective function from the $10^{t h}$ generation does not change, as exposed in Figure 4. The 16gen./20ind. case was run in order to check that the results are the same than in the $20 \mathrm{gen}$./20ind. case, justifying the number of gen./ind. used and consequently reducing the computational effort in the upcoming RBDO problem, which is very time consuming because it requires to perform several DO phases.

As can be seen, it was proven that reducing the number of individuals in this particular problem does not worsen the results whereas increasing the number of generations helps the convergence to a better optimum and consequently leads to improvements in the objective function. This gives an idea of the characteristics of the problem, which by nature has several local minima and causes that the GA can converge to different optimal designs depending on the parameters of the algorithm.

Furthermore, as the stiffened panel is pushed until collapse, the shortening when the panel fails $S h_{f, 0}$ can be obtained. It is important that the values of $S h_{f, 0}$ from the FE analyses are coherent with those of the experimental tests in order to validate the FE model for virtual testing. Moreover, $S h_{f, 0}$ is critical because it marks the hard barrier that the design can reach, making interesting to consider it as a probabilistic constraint in order to accomplish that the probability of collapse 


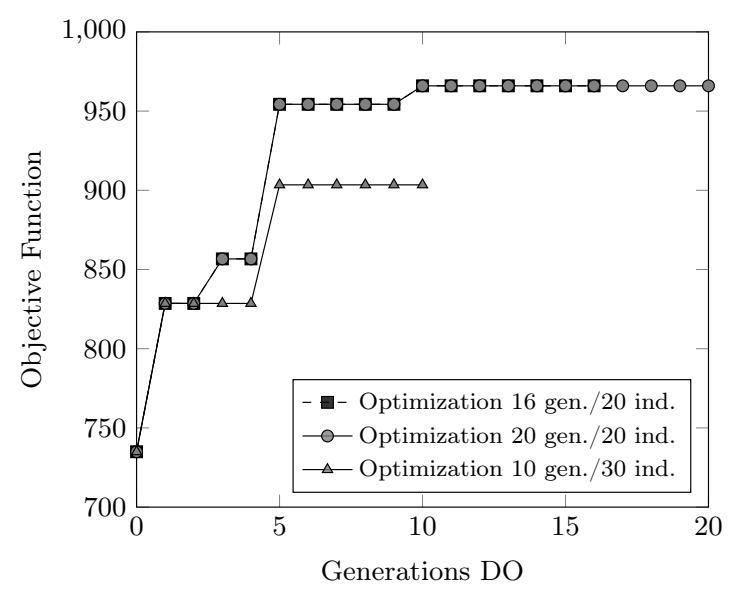

Fig. 4: Convergence of the objective function in the DO.

before $S h_{f, 0}$ is very low. The shortening of failure $S h_{f, 0}$ and the optimum layups obtained from all the DO cases are presented in Table 3.

\subsection{Formulation of the reliability-based design optimization problem}

The main difference between RBDO and DO is that the former includes within the optimization problem the uncertainty of some variables that influence the structural responses by considering them as random variables instead of fixed parameters. As a consequence some of the constraints of the optimization problem become probabilistic. A design obtained from a RBDO process assures the fulfillment of the probabilistic constraints with a desired target reliability level $\beta^{T}$ when some of the parameters involved in the structural responses are random. In contrast, a design obtained from a DO process cannot ensure the fulfillment of the constraints if some degree of uncertainty exists in the problem. Figure 5 shows an illustrative scheme where the essence of DO and RBDO can be clearly appreciated.

The general RBDO problem can be expressed mathematically as:

$$
\min F(\mathbf{d})
$$

subject to:

$$
\begin{array}{r}
g_{j}(\mathbf{d}) \leq 0 \quad(j=1, \ldots, n) \\
P\left[G_{i}(\mathbf{d}, \mathbf{x}) \leq 0\right] \leq P_{f, i} \quad(i=1, \ldots, m)
\end{array}
$$

where $F$ is the objective function, $g_{j}$ is the $j$ deterministic constraint, $G_{i}$ is the $i$ probabilistic constraint, $\mathbf{d}$ are the design variables and $\mathbf{x}$ the random variables. Additionally, $P[-]$ denotes the probability operator and $P_{f}$ is the probability of failure of the probabilistic constraint.

According to Aoues and Chateauneuf (2010), the methods employed to solve RBDO problems can be classified in three groups: 


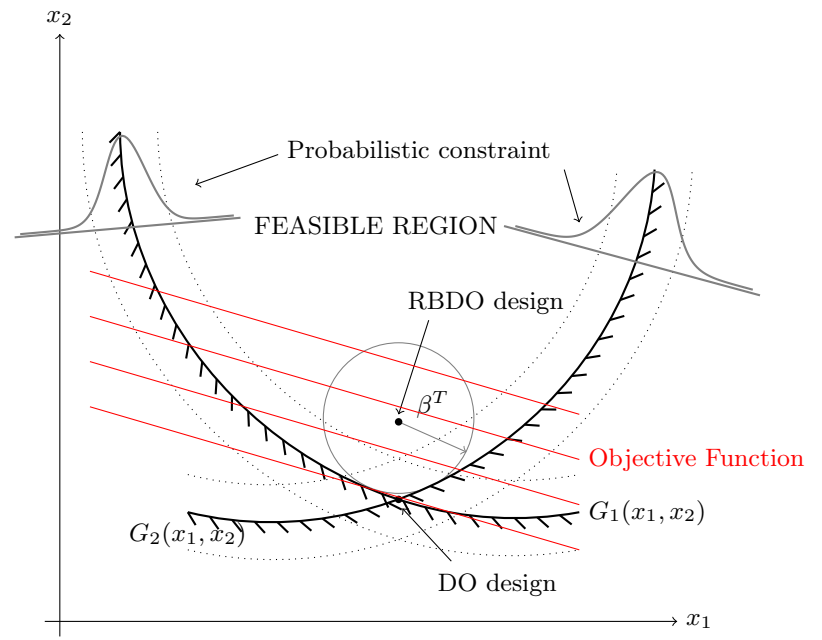

Fig. 5: Illustrative scheme of DO vs. RBDO.

1. Two level methods (i.e. Enevoldsen and Sorensen (1994), Tu et al (1999)).

2. Mono level methods (i.e. Chen et al (1997), Kuschel and Rackwitz (2000).

3. Decoupled methods (i.e. Du and Chen (2004), Cheng et al (2006)).

In this research a decoupled method based on the Sequential Optimization and Reliability Assessment (SORA, Du and Chen (2004)) is employed, being the main difference that the RA is performed relying in a global approximation model. The election of a decoupled RBDO algorithm is based on the lower computational effort when compared to other methods as they transform the RBDO problem into a sequence of $\mathrm{DO}$ and RA that are repeated until convergence, and particularly the SORA over other decoupled methods mainly for its robustness and accuracy (Aoues and Chateauneuf (2010)).

The objective of this research is to obtain the maximum reaction force $R f$ of a composite stiffened panel while being sure that the failure will happen beyond a specified value of shortening $S h_{f, 0}=6.32 \mathrm{~mm}$ when considering the uncertainty associated to the elastic properties of the composite material.

Considering this, the Reliability-Based Design Optimization (RBDO) problem can be defined as exposed in Eq. 8:

$$
\max R f
$$

subject to:

$$
P[G \leq 0] \leq P_{f}
$$

The limit-state function is defined as $G=1-\frac{S h_{f, 0}}{S h_{f}}$, where $S h_{f}$ is the shortening when the panel fails in the RBDO process and $S h_{f, 0}=6.32 \mathrm{~mm}$ is the shortening when the DO design collapses. By convention, if $G>0$ the design is safe and if $G<0$ fails, hence the design is safe when $S h_{f}>S h_{f, 0}=6.32 \mathrm{~mm}$. 
The design variables are the orientation of the layers that build the skin and stringers, like in the DO problem. The random variables are all the elastic properties of the bulk composite $\left(E_{11}, E_{22}, E_{33}, G_{12}, G_{13}\right.$ and $\left.G_{23}\right)$, following a truncated Normal distribution in order to avoid the negative values of the distribution whose statistical moments are defined in Table 4 . The assumption of considering the elastic material properties as normally distributed with a $5 \%$ coefficient of variation is based on the literature (Yang et al (2013) or Akula (2014)).

Table 4: Statistical moments of the random variables.

\begin{tabular}{llrr}
\hline Random Variable & Distribution & $\mu$ & $\sigma$ \\
\hline$E_{11}[\mathrm{MPa}]$ & Normal & 159000.0 & 7950.0 \\
$E_{22}=E_{33}[\mathrm{MPa}]$ & Normal & 10000.0 & 500.0 \\
$G_{23}[\mathrm{MPa}]$ & Normal & 5000.0 & 250.0 \\
$G_{12}=G_{13}[\mathrm{MPa}]$ & Normal & 3000.0 & 150.0 \\
\hline
\end{tabular}

The RBDO problem provides a design that maximizes the $R f$ of the panel and at the same time guarantees that it will withstand a shortening beyond $S h_{f, 0}=$ $6.32 \mathrm{~mm}$ with a established reliability $\left(1-P_{f}\right)$ when uncertainty is considered in the elastic properties of the composite material. Indeed the probability that the panel collapses before $6.32 \mathrm{~mm}$ is the probability of failure $P_{f}$ imposed by the engineer.

\section{Description of the methodology proposed}

The solution of the RBDO problem exposed in Eq. 8 requires a high computational effort for the RBDO process since the values of the objective function and constrains (both deterministic and probabilistic) are obtained through nonlinear explicit dynamic FE analyses that are performed in Abaqus Explicit 6.14.2. As stated in Section 2.1, these FE analyses include post-buckling behaviour and progressive failure considering all the possible failure modes of the fibres and the matrix of the composite.

The RBDO problem is solved through a decoupled algorithm (SORA) programmed in MATLAB (MATLAB (2013)) and which is composed of two separate steps that are performed sequentially until convergence, as shown in Figure 6.

The convergence criterion of the problem is defined as the relative difference in the reaction force of the panel $R f$ within two consecutive RBDO iterations, as expressed in Eq. 9.

$$
\frac{R f^{K+1}-R f^{K}}{R f^{K}} \leq \varepsilon
$$

where $K$ and $K+1$ are two consecutive iterations of the RBDO algorithm and $\varepsilon$ is the maximum convergence criterion value, which is set to $\varepsilon=1 \cdot 10^{-2}$. The steps required to perform the decoupled RBDO method are discussed below:

1. Deterministic Optimization (DO), which is performed through a GA. The values of the random variables in the first optimization cycle are the means 


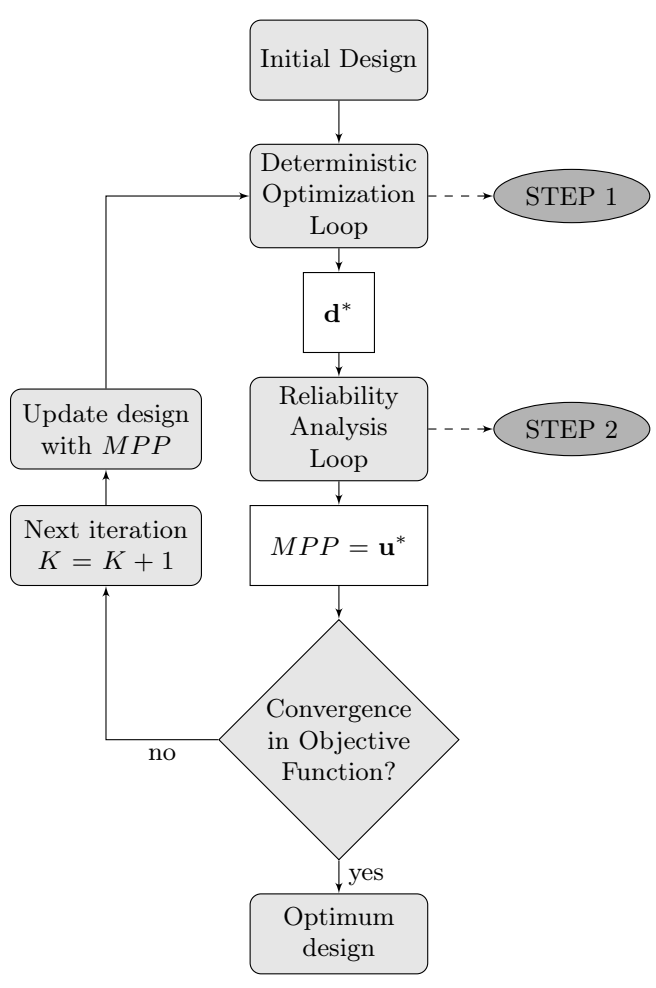

Fig. 6: Flowchart of the decoupled algorithm employed in the RBDO.

of their probability distributions. In the subsequent optimization cycles, the values of the random variables are the Most Probable failure Points (MPP) obtained in the corresponding RA step.

2. Reliability Analysis (RA), performed using the Hybrid Mean Value (HMV, Youn et al (2003)), which is an inverse MPP search algorithm that benefits from a Polynomial Chaos Expansion with Latin Hypercube Sampling (PCELHS) based metamodel (Choi et al (2004)) when the structural responses are required.

\subsection{Deterministic optimization phase}

The flowchart of a general GA is presented in Figure 7. In this research the DO problem is solved in MATLAB through the Integer ga Algorithm, which is included in the Global Optimization Toolbox and is based on the algorithms presented in Deb (2000) and Deep et al (2009). This GA attempts to minimize a penalty function instead of the objective function and employs a binary tournament selection to select individuals for upcoming generations. When all the individuals of the population are feasible the penalty function of a member is the objective function but otherwise the penalty function is the maximum objective function among 
the feasible members of the population plus a term that includes a sum of the constraint violations of the infeasible member evaluated (MATLAB (2013)).

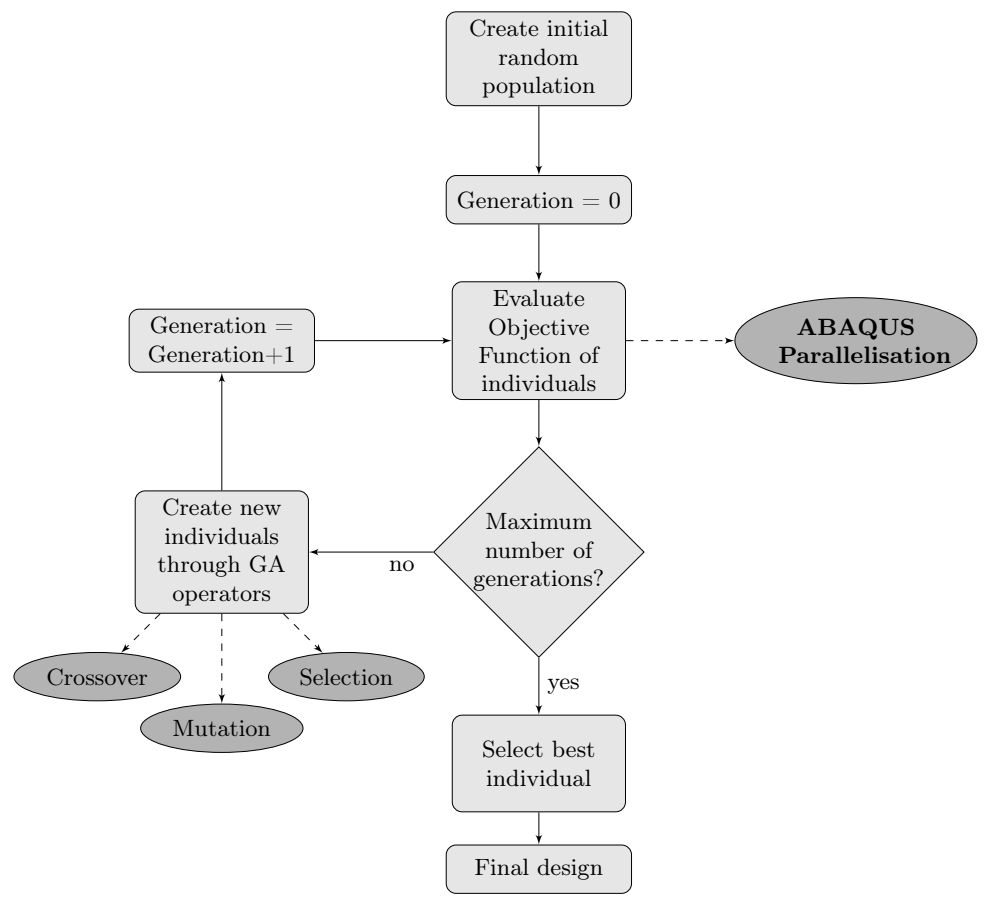

Fig. 7: Flowchart of the Genetic Algorithm (GA) employed in the Deterministic Optimization in MATLAB.

The MATLAB code generates a random initial population and makes external calls to Abaqus 6.14.2 to perform the FE simulations of the panel, which is a parallelizable task. Then the objective function and constraints obtained from those FE simulations are retrieved by MATLAB to execute the GA operators and obtain the individuals of the upcoming generation. The population size is set to 20 individuals and the maximum number of generations is 16 , which means to perform 340 runs of the FE model, all of them considering explicit nonlinear FE analyses.

\subsection{Reliability analysis phase}

\subsubsection{Formulation of the reliability analysis}

In this research the RA problem is solved by programming in MATLAB the Hybrid Mean Value (HMV, Youn et al (2003)) algorithm, which is an efficient inverse MPP search method that uses the Advanced Mean Value (AMV) for convex functions and the Conjugated Mean Value (CMV) for concave functions. The HMV requires a previous transformation of the random variables $\mathbf{x}$ into uncorrelated 
and normalized variables $\mathbf{u}$, and aims to obtain the MPP of the structure that will take part in the next DO cycle of the SORA. The MPP is defined as the most probable values that the random variables take when the limit-state function of the structure $G=0$ is reached. The distance from the MPP to the mean values of the random variables in the standard and independent space $\mathbf{u}$ is the so-called reliability index $\beta$, which is linked to the probability of failure by the following expression when the random variables follow a Normal distribution.

$$
P_{f}=\Phi(-\beta)
$$

The HMV algorithm is an iterative process that is detailed below. First the random variables $\mathbf{x}$ are transformed to the standard and independent space $\mathbf{u}$ through the general Rosenblatt tranformation. Then the limit state function $G\left(\mathbf{u}_{k}\right)$ and its gradients with respect to the random variables $\nabla G\left(\mathbf{u}_{k}\right)$ need to be calculated in order to obtain the normalized steepest direction $\boldsymbol{\alpha}_{k}$ of the limit state function through the formula:

$$
\boldsymbol{\alpha}_{k}=-\frac{\nabla_{u} G\left(\mathbf{u}_{k}\right)}{\left\|\nabla_{u} G\left(\mathbf{u}_{k}\right)\right\|}
$$

being $k$ the current iteration of the HMV algorihtm. Afterwards the upcoming point of the HMV $\mathbf{u}_{k+1}$ is obtained after recognizing if the limit state function is concave or convex. This is achieved by calculating the sign of $\xi$, which is defined as:

$$
\xi=\left(\boldsymbol{\alpha}_{k+1}-\boldsymbol{\alpha}_{k}\right) \cdot\left(\boldsymbol{\alpha}_{k}-\boldsymbol{\alpha}_{k-1}\right)
$$

If $\xi>0$, the limit-state function is convex and the upcoming point is obtained as:

$$
\mathbf{u}_{k+1}=\beta^{T} \cdot \boldsymbol{\alpha}_{k}
$$

whereas if $\xi<0$, the limit-state function is concave and the next point is obtained as:

$$
\mathbf{u}_{k+1}=\beta^{T} \cdot \frac{\boldsymbol{\alpha}_{k}+\boldsymbol{\alpha}_{k-1}+\boldsymbol{\alpha}_{k-2}}{\left\|\boldsymbol{\alpha}_{k}+\boldsymbol{\alpha}_{k-1}+\boldsymbol{\alpha}_{k-2}\right\|}
$$

During the first three iterations, the HMV calculates $\mathbf{u}_{k+1}$ through Eq. 13. This iterative process is repeated until convergence in the value of $\mathbf{u}$. After convergence the final value of $\mathbf{u}_{k+1}$ is saved as the MPP of the limit state function $\mathbf{u}^{M P P}$. The convergence of the HMV is reached when Eq. 15 is fulfilled:

$$
\frac{G\left(\mathbf{u}_{k+1}\right)-G\left(\mathbf{u}_{k}\right)}{G\left(\mathbf{u}_{k}\right)} \leq \epsilon
$$

where $k$ and $k+1$ are two consecutive iterations of the HMV algorithm and $\epsilon$ is the maximum convergence criterion value, which is set to $\epsilon=5 \cdot 10^{-3}$. Figure 8 clearly presents the steps followed by the HMV. 


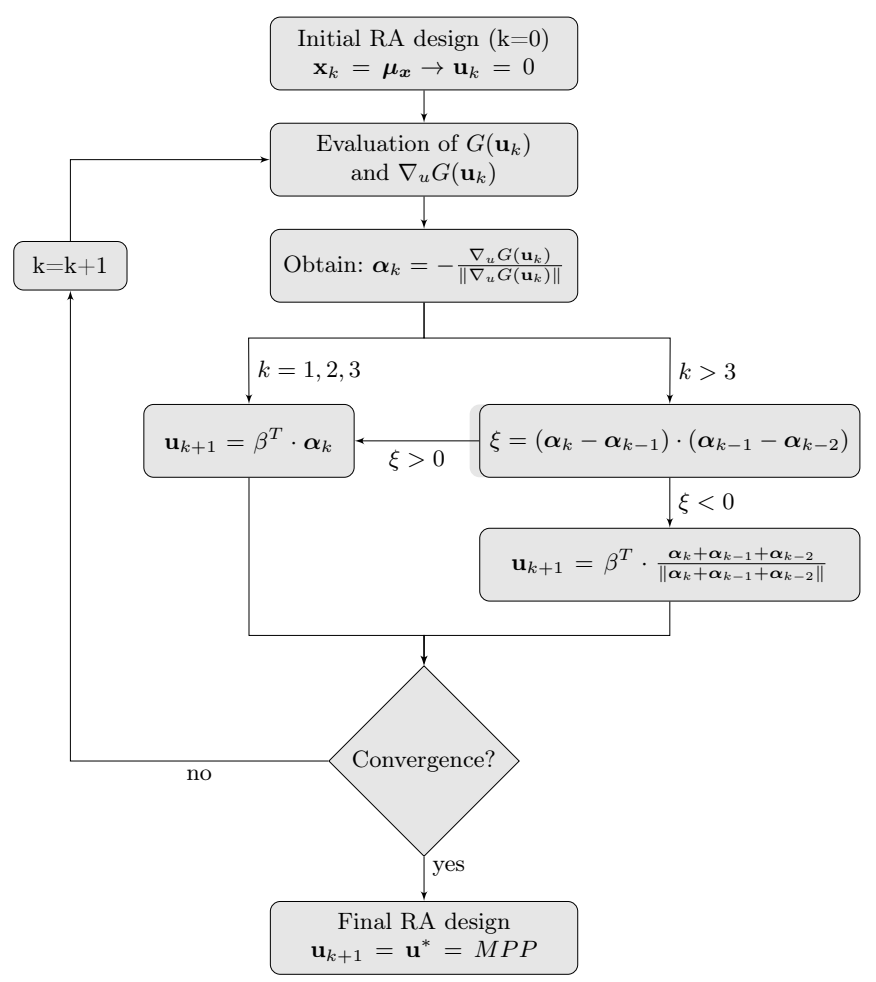

Fig. 8: Flowchart of the Reliability Analysis algorithm.

\subsubsection{Metamodel building}

When the HMV algorithm requires the evaluation of the structural responses in order to build the limit-state function $G$, it benefits from a metamodel constructed through a PCE-LHS instead of running sequentially each FE simulation. The PCE was originally derived for the spectral representation of stochastic responses in terms of Gaussian random variables in Wiener (1938). Later, other researchs (Askey and Wilson (1985) or Xiu and Karniadakis (2003)) extended the method to other non-Gaussian random variables. The PCE, whose details are exposed profusely in literature (Choi et al (2004), Eldred (2009) or Hu and Youn (2011)) is an effective choice to represent the structural responses for an uncertainty analysis as they benefit from orthogonality properties that greatly simplify the procedure of statistical calculations, such as moments (Choi et al (2007)). Moreover, the weighting functions of these orthogonal polynomials known as the Askey scheme correspond to the Probability Density Functions (PDF) of several well-known distributions, as presented in Table 5.

Particularly, the Hermite PCE for a response $R$ is an infinite polynomial expansion which in practice is truncated at a expansion order $P$ as follows:

$$
R \cong \sum_{i=0}^{P} b_{i} \cdot \Psi_{i}(\mathbf{u})
$$


Table 5: Correspondence between orthogonal polynomials and PDF

\begin{tabular}{llr}
\hline Orthogonal polynomial & PDF & Support range \\
\hline Hermite & Normal & $(-\infty,+\infty)$ \\
Legendre & Uniform & $(a, b)$ \\
Laguerre & Exponential & $(0,+\infty)$ \\
Jacobi & Beta & $(a, b)$ \\
\hline
\end{tabular}

where $b_{i}$ are the unknown coefficients and $\Psi_{i}$ are the Hermite polynomials evaluated in the normalized random variable vector $\mathbf{u}$. Usually, $\Psi_{i}(\mathbf{u})$ are multivariate polynomials that involve products of the one-dimensional Hermite polynomials $\psi_{i}\left(u_{i}\right)$, and hence the PCE includes a complete basis of polynomials up to a fixed total-order specification $P$. The one-dimensional Hermite polynomials $\psi_{i}(u)$ are generally defined as:

$$
\psi_{i}(u)=(-1)^{i} \cdot\left[\phi^{(i)}(u) / \phi(u)\right]
$$

where $\phi^{(i)}(u)$ is the $i$ th derivative of the PDF of the Normal distribution $N(0,1)$, expressed as:

$$
\phi(u)=(1 / \sqrt{2 \pi}) \exp \left(-u^{2} / 2\right)
$$

From Equation 17 the set of the $i$ th order one-dimensional Hermite polynomials can be easily derived as:

$$
\left\{\psi_{i}(u)\right\}=\left\{1, u, u^{2}-1, u^{3}-3 u, u^{4}-6 u^{2}+3, u^{5}-10 u^{3}+15 u, \ldots\right\}
$$

As an example, the multidimensional basis polynomials for a second-order expansion over two random dimensions are:

$$
\begin{aligned}
& \Psi_{0}(\mathbf{u})=\psi_{0}\left(u_{1}\right) \psi_{0}\left(u_{2}\right)=1 \\
& \Psi_{1}(\mathbf{u})=\psi_{1}\left(u_{1}\right) \psi_{0}\left(u_{2}\right)=u_{1} \\
& \Psi_{2}(\mathbf{u})=\psi_{0}\left(u_{1}\right) \psi_{1}\left(u_{2}\right)=u_{2} \\
& \Psi_{3}(\mathbf{u})=\psi_{2}\left(u_{1}\right) \psi_{0}\left(u_{2}\right)=u_{1}^{2}-1 \\
& \Psi_{4}(\mathbf{u})=\psi_{1}\left(u_{1}\right) \psi_{1}\left(u_{2}\right)=u_{1} u_{2} \\
& \Psi_{5}(\mathbf{u})=\psi_{0}\left(u_{1}\right) \psi_{2}\left(u_{2}\right)=u_{2}^{2}-1
\end{aligned}
$$

The total number of coefficients $N_{c}$ of the polynomial expansion is given by:

$$
N_{c}=1+P=\frac{(n+p) !}{n ! p !}
$$

where $n$ is the number of random variables and $p$ is the maximum order of the one-dimensional Hermite polynomials $\psi$. From this expression it can be noted that increasing the number of random variables or the order of the polynomial will cause a substantial growth in the number of terms $N_{c}$ of the PCE. Hence this will imply an appreciable increase in the sample size and consequently in the computational burden associated for complex analyses (like in this case, where post-buckling and progressive failure are involved). Therefore it is important to select carefully the random variables involved in order to spend a reasonable computational time. 
Table 6: ANOVA table for test of signification

\begin{tabular}{lrrrr}
\hline $\begin{array}{l}\text { Source of } \\
\text { variance }\end{array}$ & $\begin{array}{r}\text { Sum of } \\
\text { squares }\end{array}$ & $\begin{array}{r}\text { Degrees of } \\
\text { freedom }\end{array}$ & $\begin{array}{r}\text { Mean } \\
\text { square (MS) }\end{array}$ & $F_{0}$ \\
\hline Total Regression & $S S_{r}$ & $d f_{r}=N_{c}$ & $M S_{r}=S S_{r} / d f_{r}$ & $M S_{r} / M S_{e}$ \\
Coeff. of the Regression & $S S_{c}$ & $d f_{c}=1$ & $M S_{c}=S S_{c} / d f_{c}$ & $M S_{c} / M S_{e}$ \\
Residuals & $S S_{e}$ & $d f_{e}=N_{s}-N_{c}-1$ & $M S_{e}=S S_{e} / d f_{e}$ & \\
Total & $S S_{t}$ & $d f_{t}=N_{s}-1$ & & \\
\hline
\end{tabular}

The unknown coefficients $b_{i}$ are obtained through regression models also known as stochastic response surfaces. The polynomial expansion of Eq. 16 can be expressed in matrix notation for $N_{s}$ samples as follows:

$$
\mathbf{R}=\boldsymbol{\Psi} \mathbf{b}+\mathbf{e}
$$

where:

$$
\mathbf{R}=\left[\begin{array}{c}
R_{1} \\
R_{2} \\
\vdots \\
R_{N_{s}}
\end{array}\right], \boldsymbol{\Psi}=\left[\begin{array}{ccccc}
1 & \Psi_{1}\left(u_{1}\right) & \Psi_{2}\left(u_{1}\right) & \ldots & \Psi_{p}\left(u_{1}\right) \\
1 & \Psi_{1}\left(u_{2}\right) & \Psi_{2}\left(u_{2}\right) & \ldots & \Psi_{p}\left(u_{2}\right) \\
\vdots & \vdots & \vdots & \ddots & \vdots \\
1 & \Psi_{1}\left(u_{n}\right) & \Psi_{2}\left(u_{n}\right) & \ldots & \Psi_{p}\left(u_{N_{s}}\right)
\end{array}\right], \mathbf{b}=\left[\begin{array}{c}
b_{1} \\
b_{2} \\
\vdots \\
b_{N_{s}}
\end{array}\right], \mathbf{e}=\left[\begin{array}{c}
e_{1} \\
e_{2} \\
\vdots \\
e_{N_{s}}
\end{array}\right]
$$

being e the residuals. Usually, these regression models use the method of least squares to identify the unknown coefficients as follows:

$$
\mathbf{b}=\left(\boldsymbol{\Psi}^{T} \boldsymbol{\Psi}\right)^{-1} \boldsymbol{\Psi}^{T} \mathbf{R}
$$

Once the coefficients are obtained, the fitted model $\widehat{\mathbf{R}}$ and the residuals can be expressed as:

$$
\widehat{\mathbf{R}}=\boldsymbol{\Psi} \mathbf{b} \quad \mathbf{e}=\mathbf{R}-\widehat{\mathbf{R}}
$$

The set of $N_{s}$ samples needed to identify the unknown coefficients $\mathbf{b}$ are obtained through a LHS that requires $N_{s}$ FE simulations to obtain the set of structural responses $\mathbf{R}$. The LHS is the sampling scheme selected as it is far more efficient than the MCS method and ensures a regular distribution of the samples making that all portions of the random variables' ranges are represented.

Metamodel fitness must be checked in each particular problem to ensure the precision of the approximation (Corman and German (2010)). For PCE-based metamodels, it is usual that the test of significance of the regression is performed through ANOVA (ANalysis Of VAriance, described in Draper and Smith (1981), Montgomery and Peck (1992) or Choi et al (2007)). The ANOVA test is usually summarized in a table similar to Table 6 , where $S S_{t}, S S_{r}$ and $S S_{e}$ are given by:

$$
S S_{t}=\mathbf{R}^{T} \cdot \mathbf{R} \quad S S_{r}=\widehat{\mathbf{R}}^{T} \cdot \widehat{\mathbf{R}} \quad S S_{e}=\mathbf{e}^{T} \cdot \mathbf{e}
$$

The test statistic $F_{0}$ shows if a coefficient has a significant effect on the regression model. If $F_{0}>F_{\alpha, 1, d f e}$, where $\alpha$ denotes the $100(1-\alpha)$ th percentile of the " $F$ distribution" and $d f e$ indicates the degrees of freedom of the error in the regression, then the coefficient has a significant effect. 
In addition the metamodel fitness has been assessed through two well-known statistical metrics as the $R^{2}$ (coefficient of determination) and the RMSE (root mean square error). The $R^{2}$ is a statistic that applies a penalty for the sum of squares of error in the regression in relation to the sum of squares of the response distribution, as exposed in Eq. 26:

$$
R^{2}=1-\frac{\sum_{i=1}^{n}\left(y_{i}-\hat{y}_{i}\right)^{2}}{\sum_{i=1}^{n}\left(y_{i}-\bar{y}_{i}\right)^{2}}
$$

where $y$ is the real response point obtained from the FE simulation, $\hat{y}$ is the response point obtained from the PCE-based regression and $\bar{y}$ is the mean of all the sample response points. Therefore the range of $R^{2}$ is $(-\infty, 1]$, where the value of 1 denotes a perfect fit. The larger the $R^{2}$ estimator becomes, the better the accuracy of the metamodel is. On the other hand, the RMSE is the standard deviation of the residual distribution providing an idea of the scale error of the distribution and is inversely correlated with $R^{2}$. The formulation of the $R M S E$ is shown in Eq. 27:

$$
R M S E=\sqrt{\frac{1}{n} \sum_{i=1}^{n}\left(\frac{y_{i}-\hat{y}_{i}}{\sigma_{y}}\right)^{2}}
$$

where $\sigma_{y}$ is the standard deviation of the response distribution.

According to Choi et al (2004), the construction of a PCE-LHS metamodel involves a reasonably low number of sample points $N_{s}$, so in this research we set $N_{s}=100$. The responses of the samples are obtained through nonlinear FE simulations that are performed in parallel running Abaqus 6.14.2 in a High Performance Cluster (HPC). Afterwards the metamodel is built using a PCE approximation. Being all the 4 random variables (as $E_{22}=E_{33}$ and $G_{12}=G_{13}$, see Table 4) normally distributed, in this research a $3^{r d}$ order Hermite polynomial (Table 5) is employed. Based on the ANOVA analysis performed, a $3^{\text {rd }}$ order PCE is enough to predict the structural response properly, driving to $N_{c}=35$ coefficients in the $\mathrm{PCE}$ regression. In this case for $\alpha=0.05$ it has been proven that only 7 terms out of 35 are significant, hence raising the order of the PCE is not required (Choi et al (2004)). Besides, including higher order polynomials may lead to infeasible solutions or an excessive increase in the number of samples points without gaining accuracy.

The generation of the Hermite polynomials is programmed in a MATLAB subroutine and the regression coefficients are obtained through Eq 23. To end, ANOVA is conducted to prove that no higher order polynomials are required, and the metamodel fitness is assessed. The estimators obtained for the PCE-LHS based metamodel are $R^{2}=0.9452$ and $R M S E=0.041$, therefore it can be concluded that the approximation is accurate enough. Moreover, Figure 9 shows a plot of the real vs. PCE estimated limit-state function $G$ in order to demonstrate that the correlation between both responses is good.

Figure 10 shows the estimated PCE response of the limit state function $G$ with respect to some pairs of random variables. From this figure it can be observed that the structural response is highly influenced by the longitudinal modulus of elasticity of the material $E_{11}$ which guides the shape of the response, what is expected since the panel is loaded in compression. 


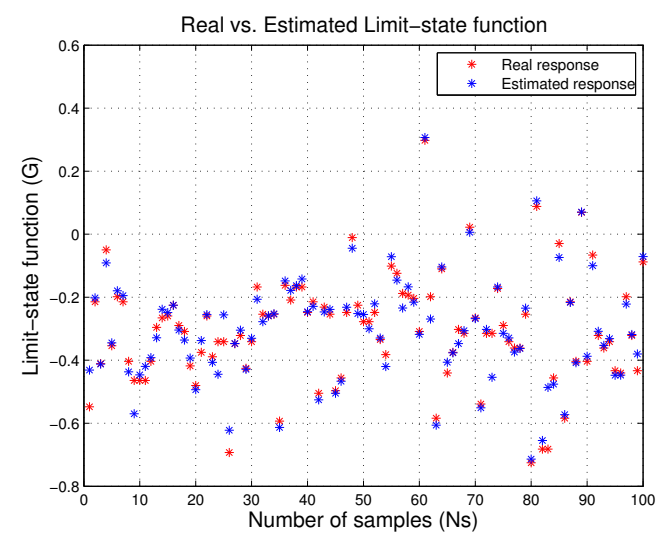

Fig. 9: Real vs. Estimated Limit-state function for 100 samples.

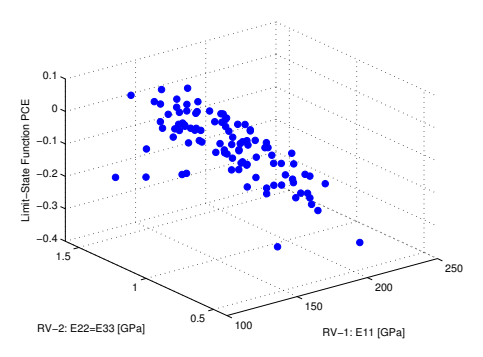

(a) Response with respect to $E_{11}$ and $E_{22}=E_{33}$

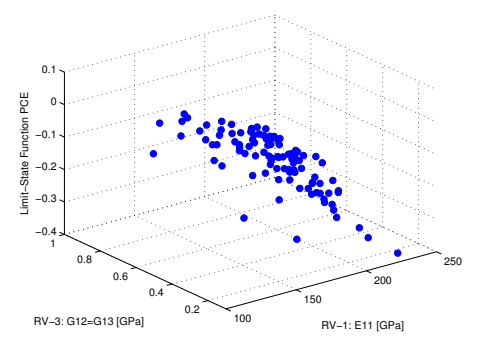

(c) Respose with respect to $E_{11}$ and $G_{12}=G_{13}$

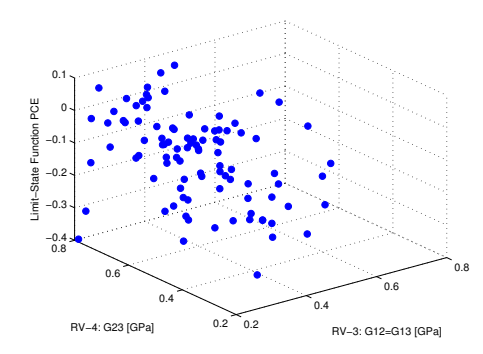

(b) Response with respect to $G_{12}=$ $G_{13}$ and $G_{23}$

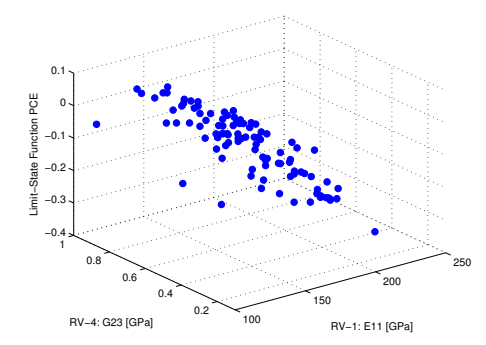

(d) Response with respect to $E_{11}$ and $G_{23}$

Fig. 10: Approximation model of the limit state function with respect to some pairs of random variables.

The flowchart of the whole RA process including sampling, metamodel building and the HMV algorithm benefiting from the PCE-LHS based metamodel is presented in Figure 11. 


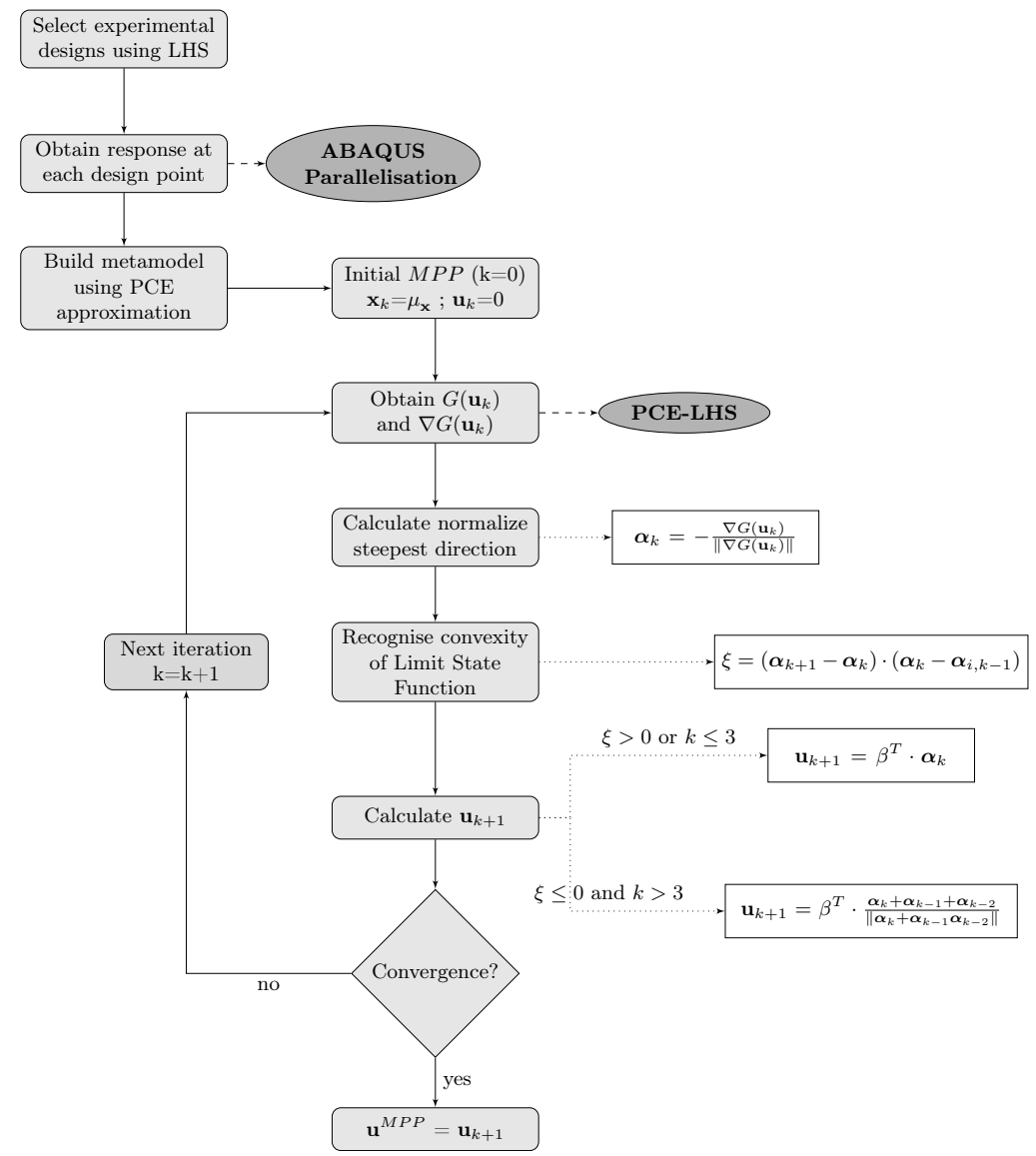

Fig. 11: Flowchart of the Reliability Analysis algorithm.

\section{Optimization results and discussion}

The DO problem defined in Eq. 6 was carried out. The results show that the GA found a new layup for maximizing the panel's reaction force $R f$. The improvements in the objective function are over a $30 \%$ compared to the initial design. Figure 12 shows the convergence of the GA.

Although the maximum number of generations was set to 16 the GA converges from the $10^{\text {th }}$ generation. The DO problem was performed in a HPC of 768 cores, a physical memory of $1.8 \mathrm{~TB}$ and a theoretical peak performance of 5.1 TFLOP's, from where we reserved 100 cores. Each FE analysis was performed using 4 cores with a global of $16 \mathrm{~GB}$ of memory. The computational effort required for solving the DO problem was of 168 hours taking a single FE analysis a average computational time of 8 hours to run. It should be noticeable that not all the simulations collapse at the same shortening so the computational effort varies from one to another. The authors have preferred not to use global approximation techniques to perform 


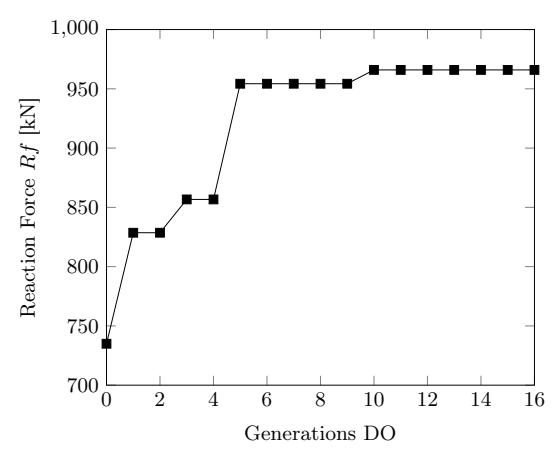

Fig. 12: Evolution of the Reaction Force (objective function) in the DO.

the DO as they are also employed in the RA step and using metamodels in both steps can detract in excess the solution of the problem.

Table 7 shows the values of the reaction force (objective function), shortening of failure, internal energy and stacking sequences of the skin and stringers before and after the deterministic optimization (DO) process. These results highlight that the optimal layup of the panel has a high number of layers oriented with $0^{\circ}$ especially on the core of the laminates, what is expected as a consequence of being loaded in the longitudinal direction and targeting to maximize the bearing capacity of the panel. Moreover, this effect is more evident in the stringers since they absorb more tensional load than the skin, as seen in figure 13 .

Table 7: Optimization results Initial design vs. DO design.

\begin{tabular}{lrr}
\hline Description & Initial design & DO design \\
\hline$R f[\mathrm{kN}]$ & 734.95 & 965.95 \\
$S h_{f}[\mathrm{~mm}]$ & 6.60 & 6.32 \\
$E n[\mathrm{~J}]$ & 2602.35 & 3402.21 \\
Skin layup & {$[45,-45,0,90] \mathrm{s}$} & {$[0,0,90,0] \mathrm{s}$} \\
Stringers layup & {$[45,0,-45,0,90] \mathrm{s}$} & {$[90,0,-45,0,0] \mathrm{s}$} \\
\hline
\end{tabular}

From the DO design the shortening when the panel collapses, which is $S h_{f, 0}=$ $6.32 \mathrm{~mm}$, is obtained. This value is imposed as the probability constraint in the RBDO problem defined in Eq. 8, whose target is to maximize the reaction force $R f$ that the panel can withstand assuring that if the elastic properties of the composite are uncertain it will collapse beyond $6.32 \mathrm{~mm}$ with a established reliability $\left(1-P_{f}\right)$. In this research two different RBDO cases were performed for reliability indexes targets of $\beta^{T}=3$ and $\beta^{T}=5$, which corresponds to probabilities of failure of $P_{f, \beta^{T}=3}=1.35 \cdot 10^{-3}$ and $P_{f, \beta^{T}=5}=2.86 \cdot 10^{-7}$ respectively. Figure 14 shows the convergence of the whole RBDO process, while Figure 15 presents the convergence of the second and third DO cycles of the SORA method.

From these figures it can be observed that the RA conducted between the second and third DO cycles converges to a similar MPP than the RA between 


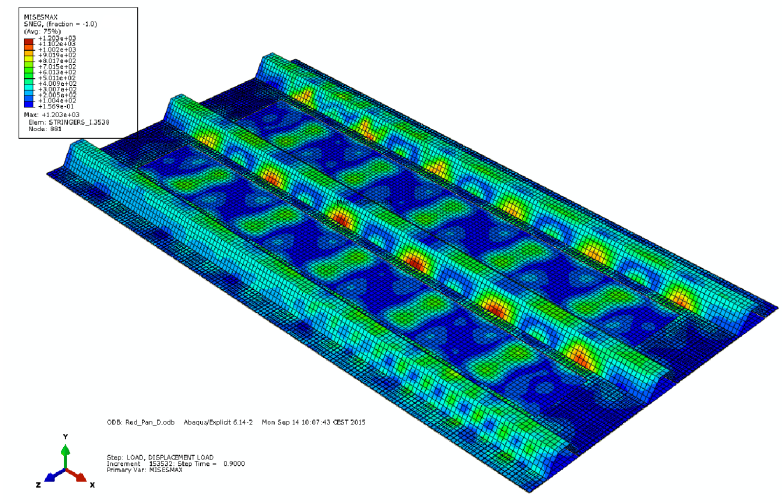

Fig. 13: Stress distribution in the stiffened panel.
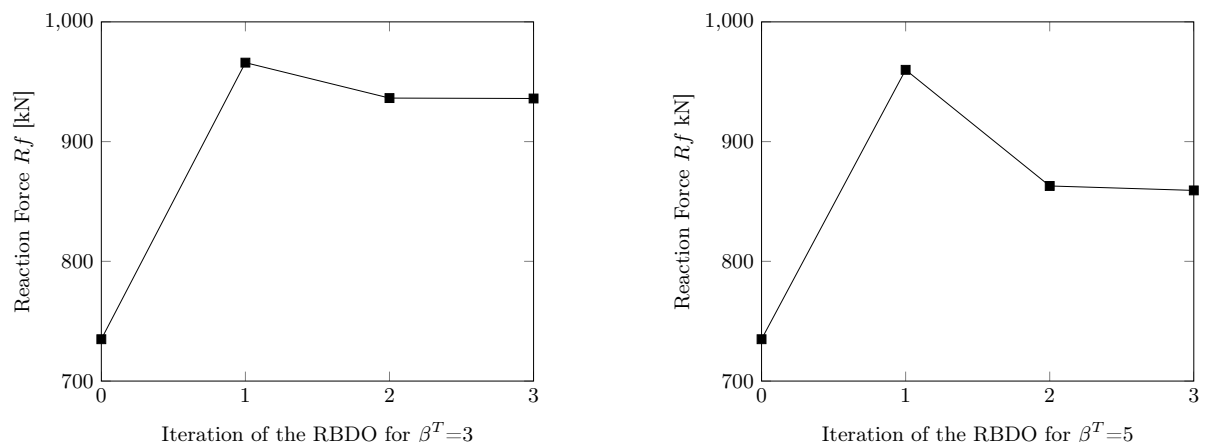

Fig. 14: Evolution of the Reaction Force (objective function) in the RBDO cases $\beta^{T}=3$ (left) and $\beta^{T}=5$ (right).

the first and second cycle and thus the third cycle starts from a design very close to the final optimum. Moreover from figure 15 it can be extracted that the GAs performed on the $2^{\text {nd }}$ and $3^{\text {rd }}$ DO cycles converge from the $10^{\text {th }}$ generation since the values of $R f$ are the same. The RBDO cases were performed in the same HPC with the same requirements for each FE analyses. Additionally, two samplings of 100 design points each were needed to perform the two RA required in the RBDO. These samplings are run in parallel, but since the number of cores reserved is 100 and each FE simulation requires 4 cores, only 25 samples are allowed to run at a time in the HPC and consequently 4 cycles of 25 samples each are required. Each cycle takes an average computational time of 8 hours (the same that a single FE simulation), so the estimated time for one RA loop is about 32 hours. In these cases the computational effort required in both RBDOs was of 514 hours for $\beta^{T}=3$ and 432 hours for $\beta^{T}=5$.

Table 8 shows the values of the reaction force (objective function), shortening of failure (probabilistic constraint), internal energy and stacking sequences of both RBDO designs compared with those of the DO design, while figure 16 shows a plot of the stacking sequence in all panels (Initial, DO and both RBDOs), where 

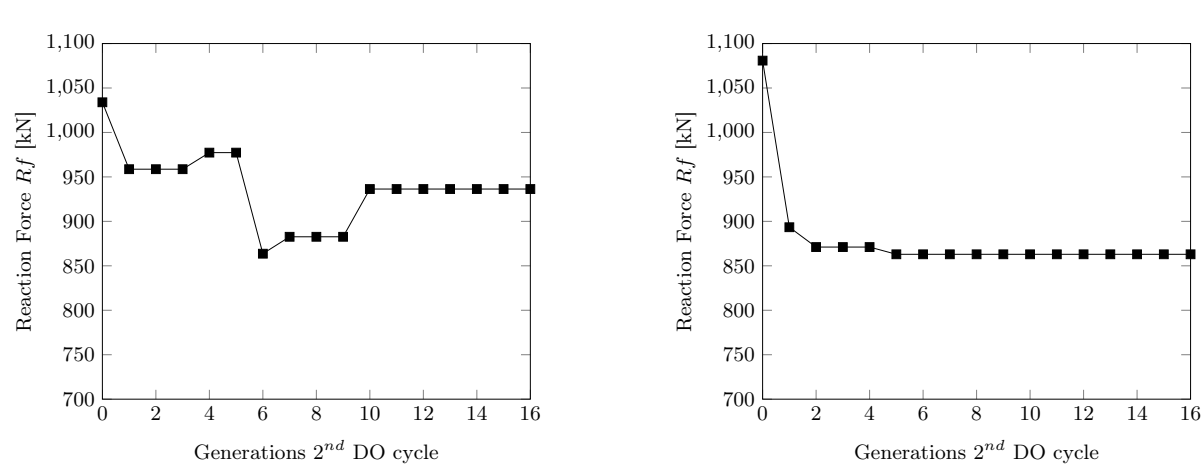

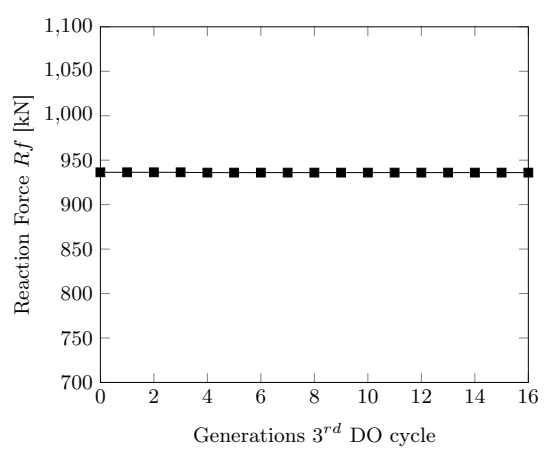

(a) $\operatorname{RBDO} \beta^{T}=3$

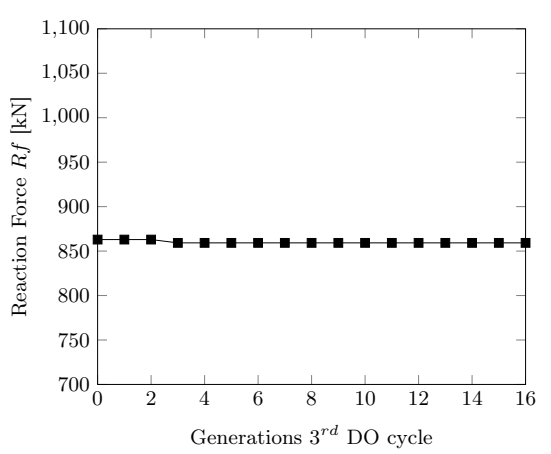

(b) $\operatorname{RBDO} \beta^{T}=5$

Fig. 15: Evolution of the Reaction Force (objective function) in the second and third DO cycle of the RBDO cases.

it can be remarked that as expected the $0^{\circ}$ oriented layers are on the inside of the stringer laminates while the $45^{\circ}$ and $-45^{\circ}$ are on the outside. The results obtained from the RBDO cases show lower values of $R f$ than the ones obtained from the DO as a consequence of including uncertainty within the optimization process, but in exchange both RBDO designs fail beyond $S h_{f, 0}$. Since the design condition $S h_{f}>6.32 \mathrm{~mm}$ needs to be accomplished in both RBDO problems with a target reliability, the RBDOs lead to designs that surpass this value of shortening although the material properties are uncertain. In return, this causes that the $R f \mathrm{~s}$ obtained from the RBDO problems are lower that the obtained from the DO problem, which is logical. The worse values in the objective function are usual in RBDO problems as they consider uncertainty in some parameters or variables, forcing the design to meet the constraints taking into account such uncertainty in the evaluation of the limit state function.

Besides it is noticeable that even though the load does not change, a significant difference in the orientation of the layers was achieved. Contrary to the DO design, the RBDO layups are more balanced as there are not too many $0^{\circ}$ oriented layers. Therefore it can be concluded that the presence of uncertainty in the material properties alters the best stacking sequence in order to accomplish the shortening limit state. 
Table 8: Optimization results RBDO designs vs. DO design.

\begin{tabular}{lrrr}
\hline Description & DO design & RBDO $\beta^{T}=3$ design & RBDO $\beta^{T}=5$ design \\
\hline$R f[\mathrm{kN}]$ & 965.95 & 936.01 & 859.24 \\
$S h_{f}[\mathrm{~mm}]$ & 6.32 & 6.41 & 6.67 \\
$E n[\mathrm{~J}]$ & 3402.21 & 3226.50 & 3050.20 \\
Skin layup & {$[0,0,90,0] \mathrm{s}$} & {$[0,0,90,45] \mathrm{s}$} & {$[90,0,45,90] \mathrm{s}$} \\
Stringers layup & {$[90,0,-45,0,0] \mathrm{s}$} & {$[90,-45,45,0,0] \mathrm{s}$} & {$[90,-45,45,0,0] \mathrm{s}$} \\
\hline
\end{tabular}

Table 9: Value of the $M P P$ of the random variables for the RBDO designs.

\begin{tabular}{lrrr}
\hline Random Variable & DO design & RBDO $\beta^{T}=3$ design & RBDO $\beta^{T}=5$ design \\
\hline$E_{11}[\mathrm{MPa}]$ & 159000.0 & 182710.0 & 198690.0 \\
$E_{22}=E_{33}[\mathrm{MPa}]$ & 10000.0 & 10061.0 & 9929.4 \\
$G_{23}[\mathrm{MPa}]$ & 5000.0 & 5064.8 & 5037.3 \\
$G_{12}=G_{13}[\mathrm{MPa}]$ & 3000.0 & 3022.8 & 2974.2 \\
\hline
\end{tabular}

Table 10: Responses of DO design vs. RBDO designs when considering the mean values of the random variables.

\begin{tabular}{lrrr}
\hline Description & DO design & RBDO $\beta^{T}=3$ design & RBDO $\beta^{T}=5$ design \\
\hline$R f[\mathrm{kN}]$ & 965.95 & 883.35 & 801.72 \\
$S h_{f}[\mathrm{~mm}]$ & 6.32 & 7.05 & 7.95 \\
$E n[\mathrm{~J}]$ & 3402.21 & 3394.40 & 3443.99 \\
\hline
\end{tabular}

Table 9 shows the MPPs obtained from the RBDO cases. The compressive effect of the load evidences that the longitudinal modulus of elasticity $E_{11}$ is the random variable by far more away from its mean value becoming the dominant of the problem.

The left side of figure 17 shows a comparison of the $R f$ responses in the initial design, the DO design and both RBDO designs for reliability indexes of $\beta^{T}=3$ and $\beta^{T}=5$ when the MPP of the random variables is considered. From this figure it can be extracted that the probabilistic constraint is somehow active in all cases, as the collapse of the optimal designs occurs near the value imposed as barrier, $S h_{f, 0}=6.32 \mathrm{~mm}$. However a more appropiate comparison between the DO and RBDO designs is carried out in the right side of figure 17. In this graph the material properties of all the designs are set to the mean values of the random variables. The values of the reaction force, shortening of failure and internal energy of the DO design compared with those of the RBDO designs considering the mean values of the random variables are shown in Table 10.

Moreover, from figure 17 it can be observed that if the material properties are set to the mean values, the $S h_{f}$ of the RBDO designs reach approximately $7 \mathrm{~mm}$ $\left(\beta^{T}=3\right)$ and $8 \mathrm{~mm}\left(\beta^{T}=5\right)$, respectively. These values are much higher than $S h_{f, 0}=6.32 \mathrm{~mm}$ as a consequence of the low value of the $P_{f}$ imposed in the RBDO problem. Indeed from the RBDO results we can state (with a reliability of $99.875 \%$ 


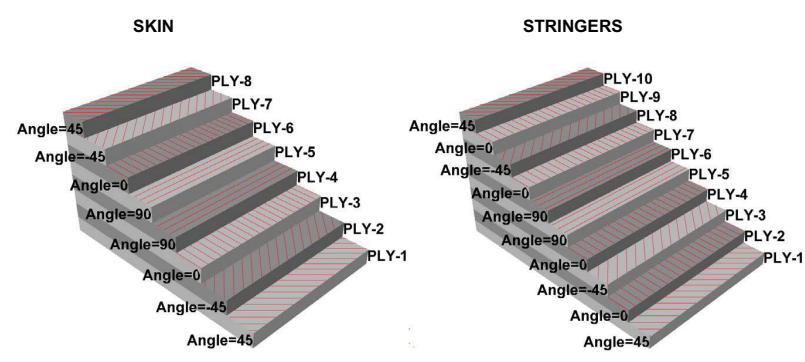

(a) Initial design
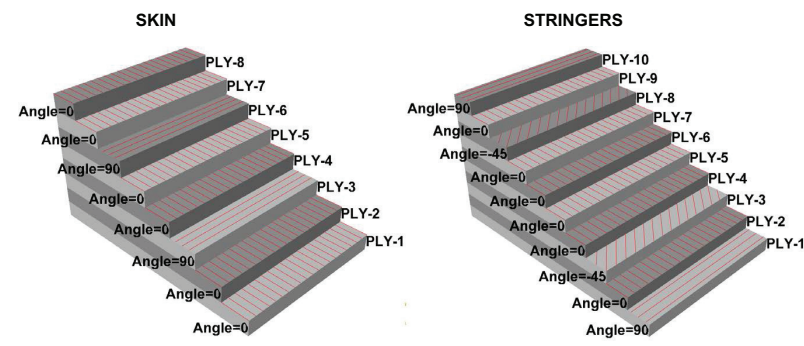

(b) DO design
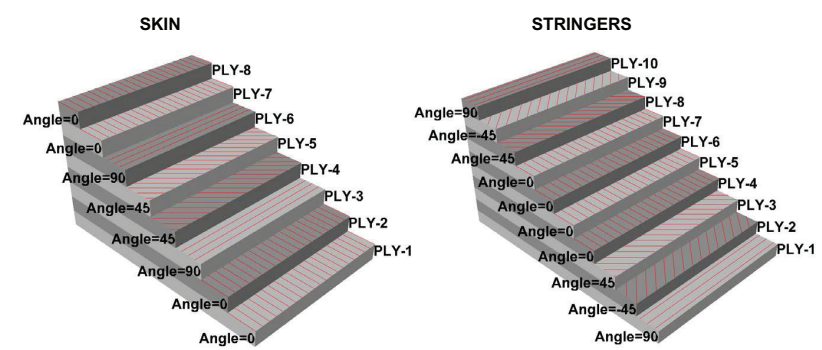

(c) $\mathrm{RBDO} \beta^{T}=3$ design
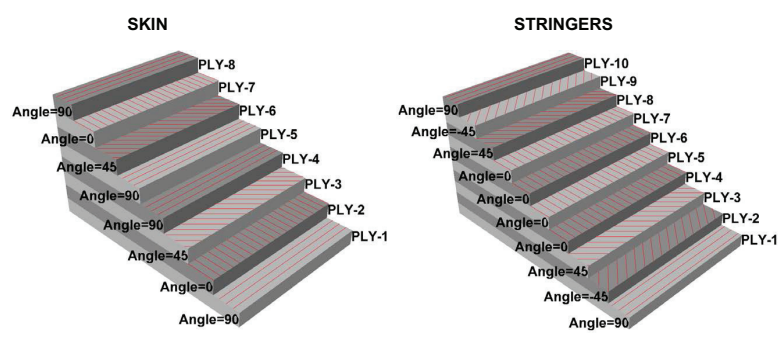

(d) RBDO $\beta^{T}=5$ design

Fig. 16: Orientation of the layers in the Initial, DO and RBDO designs. 


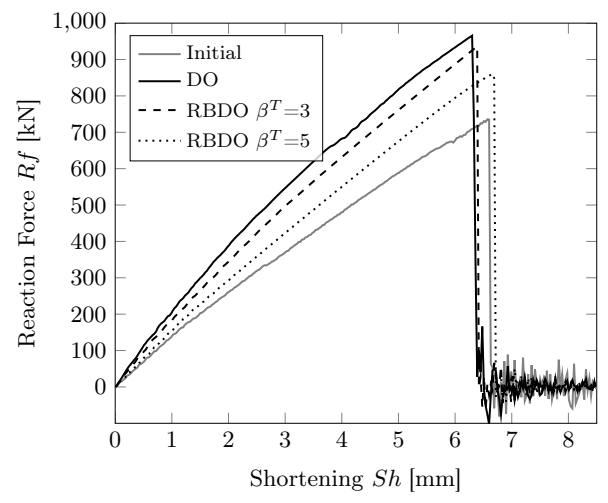

(a) Results considering MPP values

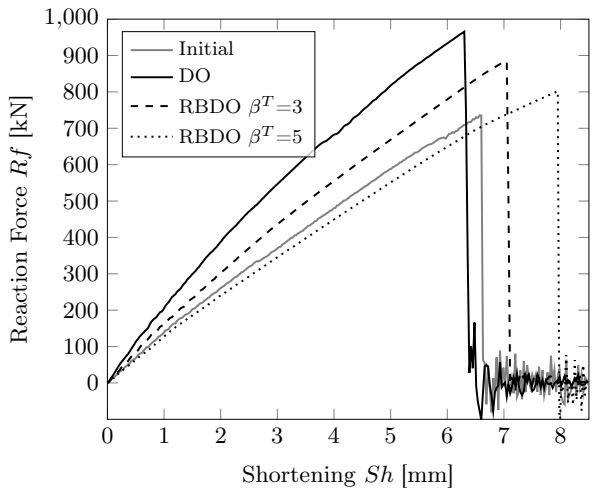

(b) Results considering mean values

Fig. 17: Response of the initial, DO, RBDO $\beta^{T}=3$ and RBDO $\beta^{T}=5$ designs considering the MPP (left) and mean (right) values of the random variables.

for $\beta^{T}=3$ and $99.9999714 \%$ for $\beta^{T}=5$ ) that the panel will collapse beyond $6.32 \mathrm{~mm}$ when considering uncertainty in the elastic properties of the composite. In other words, the $R f$ values obtained from the RBDO cases are the maximum that can be achieved if the uncertainty of the system is taken into account.

\section{Conclusions}

Composite stiffened panels are increasingly used for aeronautical applications thanks to their superior features in terms of strength, durability and weight when compared to traditional metallic or alloy panels. However, composite materials lack the well-established industrial manufacture processes of the metallics involving higher uncertainty when dealing with their mechanical properties. Thus the main aim of this research is to develop a methodology that allows performing ReliabilityBased Design Optimization (RBDO) of composite panels in post-buckling regime including progressive failure analysis and taking into account the uncertainty of the elastic properties of the composite. First, a Deterministic Optimization (DO) is performed in order to obtain the shortening of failure of the panel. This value is set as the probabilistic constraint in order to obtain a RBDO design that collapses beyond the shortening of failure. Two RBDO cases with reliability indexes $\beta^{T}=3$ and $\beta^{T}=5$ have been performed.

The DO required 340 nonlinear explicit FE analyses so as to consider the postbuckling behavior and collapse of the panel. On the other hand, both RBDOs needed to perform three DOs to converge, plus two Reliability Analyses (RA) for each RBDO case to obtain the corresponding Most Probable Failure Points (MPP). Each RA required 100 samples to construct the Polynomial Chaos Expansion with Latin Hypercube Sampling (PCE-LHS) based metamodel. In total, $1220 \mathrm{FE}$ analyses were necessary to perform both RBDO problems, which gives an idea of the high computational effort required. The authors have preferred to perform the DO without using global approximation techniques as they are also employed in 
the RA and using approximations in both the DO and RA may excessively distort the solution of the problem.

The results obtained from the RBDO show lower values of the objective function than those obtained from the DO. This is what is expected from this kind of problems as the RBDO takes into account the uncertainty of some parameters that influence the structural responses like material properties or loads, something that the DO lacks. Furthermore the probabilistic constraints in the RBDO are fulfilled and thus we can affirm that the RBDO design will meet the requirements imposed by the engineer with a high reliability.

The DO led to a final layup where the fibers were mostly oriented to $0^{\circ}$ especially on the inside of the laminate, which is expected as the load follows the same direction and the objective of the problem is to maximize the loading capacity. This fact becomes far more evident in the stringers since they absorb a larger rate of load compared to the skin. Otherwise the RBDO designs have a low number of $0^{\circ}$ oriented layers leading to a more balanced layup although the load does not change. This is due to the presence of uncertainty in the material properties of the panel. Moreover the RBDO layups assure that the design will collapse beyond the shortening of failure imposed $S h_{f, 0}$ with the target reliability defined in the RBDO problem.

Acknowledgements This research has been funded under project DPI2013-41893-R granted by the Spanish Ministry of Economy and Competitiveness. The corresponding author aknowledges the program of "Formación del Profesorado Universitario" (FPU grant AP2012-1735) from the Spanish Ministry of Education, Culture and Sports for his sponsorship. Further funding has been received by the Galician Government under grant GRC2013-056.

\section{References}

Abaqus (2014) Abaqus 6.14.2. Documentation

Acar E, Rais-Rohani M (2009) Ensemble of metamodels with optimized weight factors. Structural and Multidisciplinary Optimization 37(3):279-294

Akula VM (2014) Multiscale reliability analysis of a composite stiffened panel. Composite Structures 116:432 - 440

Aoues Y, Chateauneuf A (2010) Benchmark study of numerical methods for reliability-based design optimization. Structural and Multidisciplinary Optimization 41(2):277-294

Askey R, Wilson J (1985) Some basic hypergeometric polynomials that generalize jacobi polynomials. Memoirs of the American Mathematical Society 319, AMS, Providence, RI

Bacarreza O, Aliabadi M, Apicella A (2015) Robust design and optimization of composite stiffened panels in post-buckling. Structural and Multidisciplinary Optimization 51(2):409-422

Badalló P, Trias D, Marín L, Mayugo J (2013) A comparative study of genetic algorithms for the multi-objective optimization of composite stringers under compression loads. Composites Part B: Engineering 47:130 - 136

Bisagni C (2000) Numerical analysis and experimental correlation of composite shell buckling and post-buckling. Composites Part B: Engineering 31(8):655667 
Bisagni C, Lanzi L (2002) Post-buckling optimisation of composite stiffened panels using neural networks 58:237-247

Chen NZ, Soares CG (2007) Reliability assessment of post-buckling compressive strength of laminated composite plates and stiffened panels under axial compression. International Journal of Solids and Structures 44(22-23):7167 - 7182

Chen X, Hasselman T, Neill D (1997) Reliability based structural design optimization for practical applications. Proceedings of the 38th AIAA/ASME/ASCE/AHS/ASC Structures, Structural Dynamics, and Materials Conference Kissimee, AIAA-97-1403

Cheng G, Xu L, Jiang L (2006) A sequential approximate programming strategy for reliability-based structural optimization. Computers and Structures 84(21):1353-1367

Choi S, Grandhi R, Canfield R (2007) Reliability-based Structural Design. Springer, London, UK

Choi SK, Grandhi RV, Canfield Ra, Pettit CL (2004) Polynomial Chaos Expansion with Latin Hypercube Sampling for Estimating Response Variability. AIAA Journal 42(6):1191-1198

Cid Montoya M, Costas M, Díaz J, Romera L, Hernández S (2015) A multiobjective reliability-based optimization of the crashworthiness of a metallic-gfrp impact absorber using hybrid approximations. Structural and Multidisciplinary Optimization 52(4):827-843

Corman JA, German BJ (2010) A Comparison of Metamodeling Techniques for Engine Cycle Design Data. 13th AIAA/ISSMO Multidisciplinary Analysis Optimization Conference, 13-15 September 2010, Fort Worth, Texas

Deb K (2000) An efficient constraint handling method for genetic algorithms. Computer Methods in Applied Mechanics and Engineering 186(2-4):311-338

Deb K, Pratap A, Agarwal S, Meyarivan T (2002) A fast and elitist multiobjective genetic algorithm: NSGA-II. IEEE Transactions on Evolutionary Computation 6(2):182-197

Deep K, Singh KP, Kansal ML, Mohan C (2009) A real coded genetic algorithm for solving integer and mixed integer optimization problems. Applied Mathematics and Computation 212(2):505-518

Degenhardt R, Rolfes R, Zimmermann R, Rohwer K (2006) COCOMAT - Improved material exploitation of composite airframe structures by accurate simulation of postbuckling and collapse. Composite Structures 73(2):175-178

Díaz J, Cid Montoya M, Hernández S (2016) Efficient methodologies for reliabilitybased design optimization of composite panels. Advances in Engineering Software 93:9- 21

Draper R, Smith H (1981) Applied Regression Analysis. Wiley, New York, USA

Du X, Chen W (2004) Sequential Optimization and Reliability Assessment Method for Efficient Probabilistic Design. Journal of Mechanical Design 126(2):225-233

Eldred MS (2009) Recent Advances in Non-Intrusive Polynomial Chaos and Stochastic Collocation Methods for Uncertainty Analysis and Design. 50th AIAA/ASME/ASCE/AHS/ASC Structures, Structural Dynamics, and Materials Conference, 4-7 May 2009, Palm Springs, California

Eldred MS, Agarwal H, Perez VM, Wojtkiewicz SF, Renaud JE (2007) Investigation of reliability method formulations in DAKOTA/UQ. Structure and Infrastructure Engineering 3(3):199-213 
Enevoldsen I, Sorensen J (1994) Reliability-based optimization in structural engineering. Structural Safety 15(3):169-196

Faggiani A, Falzon BG (2007) Optimization Strategy for Minimizing Damage in Postbuckling Stiffened Panels. AIAA Journal 45(10):2520-2528

Goldberg DE (1989) Genetic Algorithms in Search, Optimization and Machine Learning, 1st edn. Addison-Wesley Longman Publishing Co., Inc., Boston, MA, USA

Hashin Z (1980) Failure Criteria for Unidirectional Fiber Composites. Journal of Applied Mechanics 47(2):329-334

Hashin Z, Rotem A (1973) A Fatigue Criterion for Fiber-Reinforced Materials. Journal of Composite Materials 7(4):448-464

Hawk J (2005) The boeing 787 dreamliner: more than an airplane. Presentation to AIAA/AAAF aircraft noise and emissions reduction symposium American institute of Aeronautics and Astronautics and Association, Aeronautique et Astronautique de France; 25/05/2005

Hernandez S, Diaz J, Baldomir A, Cid M, Lopez C (2013) Comparison of reliability based structural optimization methodologies in the design of aircraft structures. Safety, Reliability, Risk and Life-Cycle Performance of Structures and Infrastructures - International Conference on Structural Safety and Reliabiliy, ICOSSAR 2013 pp 4943-4949

Holland JH (1992) Adaptation in Natural and Artificial Systems: An Introductory Analysis with Applications to Biology, Control and Artificial Intelligence. MIT Press, Cambridge, MA, USA

Hu C, Youn BD (2011) Adaptive-sparse polynomial chaos expansion for reliability analysis and design of complex engineering systems. Structural and Multidisciplinary Optimization 43:419 - 442

Irisarri FX, Laurin F, Leroy FH, Maire JF (2011) Computational strategy for multiobjective optimization of composite stiffened panels. Composite Structures 93(3):1158-1167

Jin R, Chen W, Simpson T (2001) Comparative studies of metamodelling techniques under multiple modelling criteria. Structural and Multidisciplinary Optimization 23(1):1-13

Karadeniz H, Togan V, Vrouwenvelder T (2009) An integrated reliability-based design optimization of offshore towers. Reliability Engineering and System Safety 94(10):1510-1516

Kusano I, Baldomir A, Ángel Jurado J, Hernández S (2015) Probabilistic optimization of the main cable and bridge deck of long-span suspension bridges under flutter constraint. Journal of Wind Engineering and Industrial Aerodynamics 146:59-70

Kuschel N, Rackwitz R (2000) Optimal design under time-variant reliability constraints. Structural Safety 22(2):113-127

Lanzi L, Giavotto V (2006) Post-buckling optimization of composite stiffened panels: Computations and experiments. Composite Structures 73(2):208-220

Lapczyk I, Hurtado JA (2007) Progressive damage modeling in fiber-reinforced materials. Composites Part A: Applied Science and Manufacturing 38(11):2333 $-2341$

Leheta H, Mansour A (1997) Reliability-based method for optimal structural design of stiffened panels. Marine Structures 10(5):323-352 
Marín L, Trias D, Badalló P, Rus G, Mayugo J (2012) Optimization of composite stiffened panels under mechanical and hygrothermal loads using neural networks and genetic algorithms. Composite Structures 94(11):3321-3326

Marsh G (2007) Airbus takes on Boeing with reinforced plastic A350 XWB. Reinforced Plastics 51(11):26-29

MATLAB (2013) MATLAB R2013a Documentation

Montgomery D, Peck E (1992) Introduction to Linear Regression Analysis. Wiley, New York, USA

Ostergaard M, Ibbotson A, Roux O, Prior A (2011) Virtual testing of aircraft structures. CEAS Aeronautical Journal 1(1-4):83-103

Qu X, Haftka R (2003) Reliability-based design optimization of stiffened panels. In: Uncertainty Modeling and Analysis, 2003. ISUMA 2003. Fourth International Symposium on, pp 292-297

Rognin F, Zhang H, Abdi F (2010) Robust Design of Composite Stiffened Panels under Discrete Source Damage, a Combined Durability- Reliability Evaluation. 51st AIAA/ASME/AHS/ACS Structures, Structural Dynamics and Materials Conference, Orlando, Florida (AIAA 2010-3094):1-13

Shabana Y, Elsawaf A (2015) Nonlinear multi-variable optimization of layered composites with nontraditional interferences. Structural and Multidisciplinary Optimization 42:171-184

Simpson T, Poplinski J, Koch NP, Allen J (2014) Metamodels for computer-based engineering design: Survey and recommendations. Engineering with Computers 17(2):129-150

Sinha K (2007) Reliability-based multiobjective optimization for automotive crashworthiness and occupant safety. Structural and Multidisciplinary Optimization 33(3):255-268

Stevens K, Ricci R, Davies G (1995) Buckling and postbuckling of composite structures. Composites 26(3):189-199

Todoroki A, Sekishiro M (2008) Modified efficient global optimization for a hatstiffened composite panel with buckling constraint. AIAA Journal 46(9):22572264

Tu J, Choi KK, Park YH (1999) A New Study on Reliability-Based Design Optimization. Journal of Mechanical Design 121(4):557-564

Wiener N (1938) The Homogoeneous Chaos. American Journal of Mathematics 60(4):897-936

Xiu D, Karniadakis GE (2003) The Wiener-Askey polynomial chaos for stochastic differential equations. SIAM Journal of Scientific Computing 187:137-167

Xu YJ, You T (2013) Minimizing thermal residual stresses in ceramic matrix composites by using Iterative MapReduce guided particle swan optimization algorithm. Comosite Structures 99:388 - 396

Xu YJ, Zhang WH, Camoret D, Domaszewski M (2012) Minimizing thermal residual stresses in $\mathrm{C} / \mathrm{SiC}$ functionally graded material coating of $\mathrm{C} / \mathrm{C}$ Composites by using particle swan optimization algorithm. Computational Materials Science 61:99- 105

Yang N, Das P, Blake J, Sobey A, Shenoi R (2013) The application of reliability methods in the design of tophat stiffened composite panels under in-plane loading. Marine Structures 32:68-83

Youn B, Choi K, Park Y (2003) Hybrid analysis method for reliability-based design optimization. Journal of Mechanical Design 125(2):221-232 
Youn B, Choi K, Yang RJ, Gu L (2004) Reliability-based design optimization for crashworthiness of vehicle side impact. Structural and Multidisciplinary Optimization 26(3-4):272-283

Zhao YG, Ono T (2001) Moment methods for structural reliability. Structural Safety 23(1):47-75

Zimmermann R, Rolfes R (2006) POSICOSS - Improved postbuckling simulation for design of fibre composite stiffened fuselage structures. Composite Structures $73(2): 171-174$ 